\title{
ENERGÍA EÓLICA Y PAISAJE. IDENTIFICACIÓN Y CUANTIFICACIÓN DE PAISAJES AFECTADOS POR INSTALACIONES EÓLICAS EN ANDALUCÍA
}

\author{
María del Pilar Díaz Cuevas \\ Alfonso Fernández Tabales \\ María Fernanda Pita López \\ Departamento de Geografía Física y Análisis Geográfico Regional. Universidad de Sevilla. \\ pilard@us.es, aftabales@us.es,mfpita@us.es
}

\section{RESUMEN}

Este artículo analiza la distribución territorial y paisajística de los parques eólicos en Andalucía, identificando y cuantificando las superficies afectadas por cambios paisajísticos como consecuencia de la implantación de estas infraestructuras. Ello se lleva a cabo a partir de la generación de una geodatabase de energías renovables que recoge información espacial detallada de estas infraestructuras, con un nivel de precisión hasta el momento inédito en Andalucía. El uso de las capacidades analíticas de los Sistemas de Información Geográfica permite la definición y generación de nuevos indicadores paisajísticos relacionados con las instalaciones de energía eólica, no aplicados previamente para la Comunidad Autónoma Andaluza.

Palabras clave: energía eólica, territorio, paisaje, geodatabase, Sistemas de Información Geográfica.

\section{ABSTRACT}

This article analyzes the spatial and landscape distribution of wind farms in the region of Andalusia, Spain, and identifies and quantifies the surface area affected by landscape changes resulting from the implementation of this type of infrastructure. This is done by creating a geodatabase of renewable energies that includes detailed spatial information of these infrastructures with a scale of accuracy that is unprecedented in Andalusia. By using

Fecha de recepción: octubre 2014.

Fecha de aceptación: julio 2015. 
the analytical abilities of Geographical Information Systems it has been possible to define and create new landscape indicators linked to the installation of wind farms that have not been applied previously in the Andalusian Autonomous Community.

Keywords: wind energy, territory, landscape, geodatabase, Geographical Information Systems.

\section{INTRODUCCIÓN. PAISAJE, ENERGÍAS RENOVABLES Y VALORACIÓN SOCIAL}

La preocupación actual por la degradación de los paisajes y la demanda social creciente de paisajes de calidad, ha puesto de manifiesto la necesidad de protección, ordenación y gestión de los mismos, así como de mejora de la regulación de las actuaciones que sobre ellos tienen lugar. Esta realidad se plasma en la elaboración por parte del Consejo de Europa del Convenio Europeo del Paisaje ${ }^{1}$ (en adelante CEP), ratificado por España en 2008. Entre los avances más destacados recogidos en este documento se encuentra la propia definición de paisaje, como «cualquier parte del territorio tal como es percibida por las poblaciones, cuyo carácter resulta de la acción de factores naturales y/o humanos y de sus interrelaciones»; afianzando la consideración de que todo el territorio es paisaje, incluso el más cotidiano, y como tal debe ser valorado y administrado, ampliando así la tendencia a estimar sólo los paisajes excepcionales.

En Andalucía, la importancia del paisaje en la región viene recogida en su Estatuto de Autonomía ${ }^{2}$, que destaca el derecho de la población a su disfrute en sus artículos 10, 28 y 33; a ello se suma el incremento del número y calidad de los instrumentos y actuaciones de la política territorial, agraria, ambiental o de patrimonio que contienen manifestaciones de sensibilidad y aprecio hacia los paisajes, actuaciones que por el momento culminan con la aprobación de la Estrategia del Paisaje de Andalucía ${ }^{3}$.

En relación a las energías renovables, las connotaciones positivas asociadas a las mismas hacen que por lo general dispongan en España de una valoración ambiental, política y social positiva (ASIF, 2007). Ello se debe a que permiten una reducción de la elevada dependencia energética y una disminución en la emisión de Gases de Efecto Invernadero, uno de los principales vectores de actuación en la lucha ante el cambio climático. A este respecto, según los últimos datos disponibles, derivados de la Agencia Andaluza de la Energía (2012), el grado de autoabastecimiento energético de ésta se situó en 2012 en el 13,6\%, frente al 5,8\% de 2006, del que un $98,1 \%$ posee origen renovable; y el aporte de las energías renovables a la estructura energética primaria fue del $14,4 \%{ }^{4}$, siendo la energía eólica la que presenta la mayor potencia instalada (en torno a 3252MW distribuidos en 144 parques eólicos diferentes).

Por otro lado, se abren camino concepciones según las cuales estas energías podrían constituir auténticas oportunidades para fomentar un desarrollo territorial endógeno y más equilibrado (Díaz, Rodríguez y Zoido, 2010). Así, la implantación de parques eólicos en áreas rurales con escaso desarrollo económico supone una apreciable fuente de ingresos para los municipios, que los ayuntamientos obtienen con las licencias de obras, impuesto

1 Convenio Europeo del Paisaje, 20 de noviembre de 2000. Florencia.

2 Ley Orgánica 2/2007, de 19 de marzo, de reforma del Estatuto de Autonomía de Andalucía.

3 Estrategia del Paisaje de Andalucía, Boletín Oficial de la Junta de Andalucía n ${ }^{\circ}$ 62, 6 de marzo de 2012.

4 El objetivo planteado en el PASENER (Plan Andaluz de Sostenibilidad Energética) para 2013 era el $17 \%$ 
de actividades económicas y con la renta percibida cuando los aerogeneradores se instalan en terrenos de propiedad municipal; asimismo los habitantes de estos territorios, además de alquilar sus tierras a las empresas con este fin, pueden encontrar empleo en su construcción y, tras ésta, en su mantenimiento (Espejo y García, 2012).

A pesar de estas connotaciones positivas asociadas a este tipo de energía, su rápido ritmo de implantación, su excesiva concentración y su competencia con otros usos del suelo han derivado en numerosos conflictos relacionados con su desarrollo, vinculados principalmente con la localización de estas infraestructuras y sus implicaciones paisajísticas, generándose en el caso de España fuertes críticas a implantaciones concretas (Frolova y Pérez, 2008).

En el caso de las turbinas eólicas, la preocupación sobre las implicaciones de éstas en el paisaje es tal que incluso en la VI Conferencia del Consejo de Europa sobre la Convención Europea del Paisaje, en mayo de 2011, se presentó un informe titulado «Landscape and wind turbines» que tenía por objetivo generar una línea de actuación general para los Estados miembros, con el fin de alcanzar la integración del desarrollo de energía eólica en la dimensión paisajística, sin definir una metodología fija, de manera que pudiese ser aplicada en todos los Estados.

Descendiendo a la escala autonómica, la ya citada Estrategia del Paisaje de Andalucía identifica las energías renovables como nuevos retos paisajísticos derivados de las tendencias evolutivas y transformaciones más recientes en el territorio andaluz, y prevé para el futuro una mayor presión de éstas sobre el suelo rústico que, en ausencia de la aplicación de criterios paisajísticos, puede traducirse en un incremento del deterioro de los paisajes comunes.

\section{OBJETIVOS}

Los objetivos de este artículo consisten en analizar la distribución paisajística de los parques eólicos en Andalucía e identificar aquella superficie del territorio sometida a cambios paisajísticos como consecuencia de la implantación de estas infraestructuras, dando así respuesta en lo referente a esta temática a las estipulaciones del artículo VI de la Convención Europea del Paisaje (necesidad de la identificación de paisajes y de las principales afecciones producidas en éstos).

Como objetivos complementarios se procederá a detallar y valorar el ritmo de implantación de estas infraestructuras en Andalucía, así como su grado de concentración en determinados espacios y su presencia en espacios naturales protegidos. Finalmente, como objetivo instrumental se plantea la utilidad de generar una geodatabase relativa a la temática, buscando aprovechar al máximo la alta precisión geométrica de las fotografías aéreas y las posibilidades de trabajo que ofrece un entorno SIG (repositorio de datos geográficos, servicios interoperables, análisis espacial, etc.).

\section{MÉTODO Y FUENTES}

\section{III.1. Generación de una geodatabase de energías renovables para Andalucía}

Todo estudio centrado en la relación entre energías renovables y territorio, independientemente de cuál sea su objetivo (evolución de la implantación, impacto paisajístico, etc.), requiere de una precisa información de detalle que permita tener identificada la localización de estas infraestructuras sobre el territorio objeto de estudio. 
Para el análisis de la implantación y distribución espacial de las instalaciones de energía renovable se ha generado una geodatabase de energías renovables en Andalucía. Ello se ha llevado a cabo mediante fotointerpretación y digitalización de la información espacial vinculada a la localización de aerogeneradores y plantas solares a partir de las series de ortofotografías aéreas existentes en la región (Tabla 1). Éstas, de resolución métrica y submétrica, se encuentran publicadas como Web Mapping Services (WMS) en el geoportal de la Infraestructura de Datos Espaciales de Andalucía.

Tabla 1

RELACIÓN DE SERIES DE ORTOFOTOGRAFÍAS UTILIZADAS

\begin{tabular}{|l|c|c|}
\hline \multicolumn{1}{|c|}{ Año } & Resolución $(\mathbf{m})$ & Tipo \\
\hline $1984-1985$ & 1 & Blanco y Negro \\
\hline 1998 & 0,5 & Blanco y Negro \\
\hline $2001-2002$ & 0,5 & Color \\
\hline $2004-2005$ & 0,5 & Color \\
\hline 2007 & 0,7 & Color \\
\hline 2009 & 0,5 & Color \\
\hline 2011 & 0,5 & Color \\
\hline
\end{tabular}

Fuente: Elaboración propia a partir de la IDE-Andalucía.

En una primera etapa, se convirtió el dato bruto ráster de las ortofotografías en información vectorial interpretada mediante fotointerpretación y digitalización de la información existente en ellas. Posteriormente, toda esta información espacial ha sido completada con información temática procedente de la Agencia Andaluza de la Energía, la Asociación Empresarial Eólica y la Asociación de la Industria Fotovoltaica, que caracterizan cada planta en función de su potencia instalada y otras informaciones de interés (número y modelo de aerogeneradores por parque eólico, promotor, etc.). Por último se han añadido datos relativos a la localización y potencia instalada de centrales de biomasa eléctrica y minihidráulica, como complemento de la información anterior. Toda esta información (la de partida y la originada mediante digitalización), se ha incorporado a una base de datos geográfica o geodatabase, cuya utilización implica que mediante la construcción de sencillas sentencias SQL (Structure Query Language), fácilmente reutilizables en actualizaciones futuras, hayan podido generarse relaciones de datos de manera virtual, de forma dinámica, que son actualizadas automáticamente y permiten generar una amplia gama de indicadores para las energías renovables.

Cabe destacar que la digitalización de aerogeneradores (círculos en el ejemplo de la Figura 1) y plantas solares y la posterior asociación de información temática constituye un aspecto clave. Ello se debe a que, hasta ahora, la única información existente sobre la localización de parques eólicos y centrales solares se correspondía con información vectorial incluida en la publicación digital «Datos Espaciales de Andalucía para escalas intermedias» (Instituto de Estadística y Cartografía de Andalucía, 2013), que incluye una capa espacial que representaba el punto central de cada parque eólico (triángulos negros en la Figura 1) y de cada instalación solar, pero no cada aerogenerador singularizado ni la superficie real de cada planta solar. 
De esta forma, la geodatabase elaborada en esta investigación representa un notable avance instrumental, ya que el número de aerogeneradores implantados o la superficie ocupada por las centrales solares para cualquier ámbito espacial son variables fácilmente extraídas de la base de datos y potencialmente utilizables para la generación de indicadores. Así, el nivel de detalle proporciona una gran flexibilidad en la selección del ámbito espacial para el cálculo de los indicadores que podrían ir desde la escala autonómica, a niveles intermedios (municipios, comarcas, espacios protegidos, unidades de paisaje, etc.) e incluso a nivel de parque eólico o solar individual.

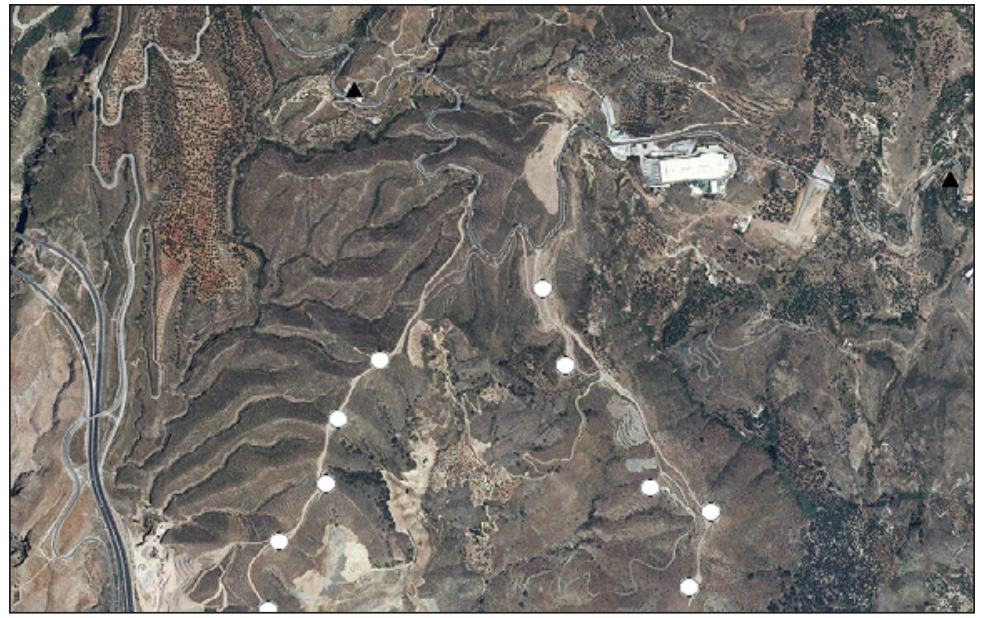

Fuente: elaboración propia.

\section{III.2. Utilización de las caracterizaciones paisajísticas existentes para la Comunidad Autónoma Andaluza}

Además de contar con la base de datos espacial de energías renovables en Andalucía, se han utilizado las dos caracterizaciones paisajísticas previamente existentes para el ámbito de estudio; el Mapa de los paisajes de Andalucía y el Atlas de los paisajes de España, los cuales recogen la amplia diversidad paisajística de la región y han servido como base para la delimitación de ámbitos de análisis en la implantación de instalaciones energéticas.

\section{III.2.1. Mapa de los paisajes de Andalucía}

Elaborado por las Consejerías de Medio Ambiente y de Obras Públicas y Transportes de la Junta de Andalucía en el año 2005, el Mapa de los paisajes de Andalucía constituye un primer inventario y clasificación de los recursos paisajísticos de la región, así como una base geográfica y cartográfica imprescindible para el estudio, evaluación y tratamiento de los paisajes andaluces. 
En un primer nivel, mediante la interpretación de imágenes de satélite a escala 1:100.000 y aunando criterios de observación (homogeneidad de colores, texturas y estructuras) junto con otros que se vinculan a aspectos socioculturales y de ordenación del territorio, se delimitaron 81 ámbitos paisajísticos diferentes para la Comunidad Autónoma, con un marcado carácter morfoestructural y natural, pero también histórico, socio económico y de organización territorial.

Estos ámbitos paisajísticos se han agrupado posteriormente en el Mapa en 19 áreas paisajísticas que responden a los grandes conjuntos de morfología y usos del suelo, y que, a su vez, pueden agruparse en cinco grandes categorías de paisaje: (1) serranías, (2) campiñas, (3) altiplanos y subdesiertos esteparios, (4) valles, vegas y marismas y (5) litoral. A través de estas áreas y categorías es posible caracterizar los distintos ámbitos atendiendo a sus rasgos geomorfológicos y paisajísticos más significativos.

En un segundo nivel, se realiza un análisis de variables de índole física o cultural y una reinterpretación de los usos y cubiertas vegetales que permiten la definición de 35 unidades fisionómicas de paisaje, relacionadas fundamentalmente con las características escénicas del paisaje, responsables, en última instancia, de las características formales (textura, colores,...) y de las más variadas morfologías estructurantes (mesas, bad lands,...).

\section{III.2.2. Atlas de los paisajes de España}

En el caso del Atlas de los Paisajes de España (Mata y Sanz (dir.) 2004), éste forma parte de un proyecto transnacional para caracterizar e identificar los paisajes de la Península Ibérica e islas, y tiene como objetivo la representación de todo el territorio español a través de una cartografía continua y con idénticos criterios de caracterización del paisaje, constituyendo así la primera síntesis del paisaje del territorio español.

El Atlas identifica unidades de paisaje diferenciadas para la medición de la diversidad, tipos de paisaje como unidad intermedia (conjuntos de paisajes de parecida configuración natural e historia territorial), y asociaciones de tipos de paisaje, como unidad mayor (reproducen la imagen física de los grandes ámbitos paisajísticos, con sus formas más evidentes y los rasgos climáticos e hidrológicos fundamentales).

Así, para la Península Ibérica se identifican 1.263 unidades de paisaje, 116 tipos de paisaje y 34 asociaciones de tipos de paisaje, mientras que para el caso andaluz identifica 13 asociaciones de tipos de paisaje, que agrupan un total de 27 tipos de paisaje y éstos a su vez agrupan a 187 unidades de paisaje.

Como ya se ha señalado, estas dos experiencias de delimitación y caracterización paisajística se utilizarán como base territorial para el establecimiento de ámbitos de análisis en la interrelación energías renovables-territorio.

\section{DISTRIBUCIÓN PAISAJISTICA DE LAS INSTALACIONES DE ENERGÍA EÓLICA EN ANDALUCÍA}

Para el análisis de la distribución paisajística de las instalaciones de energía eólica en Andalucía, se han utilizado los datos de la citada geodatabase de energías renovables de Andalucía. Así, para la última serie de ortofotografías disponible, la Ortofotografía Básica Color de Andalucía 2010-2011, elaborada a partir de los vuelos realizados en los meses de 
verano de los años 2010 (mitad sur de la región) y 2011 (mitad norte), la región poseía un total de 2992,48 MW de potencia eólica instalada, repartidas en un total de 1954 aerogeneradores pertenecientes a 129 parques eólicos diferentes.

Si bien el uso de la geodatabase puede denotar cierta desactualización con respecto a los últimos datos disponibles ${ }^{5}$, permite contar con el número y localización exacta de cada aerogenerador, de gran importancia para el análisis paisajístico, por lo que parece justificada su utilización en esta investigación.

Utilizando como ámbitos de referencia las diferentes unidades definidas en el Mapa de los paisajes de Andalucía, se observa como la potencia eólica instalada se localiza a lo largo de las cinco categorías paisajísticas definidas para la región (Figura 2); siendo las Campiñas las que aglutinan los mayores valores de potencia eólica instalada $(31 \%)$, seguidas de las Serranías, los Altiplanos y Subdesiertos Esteparios y el Litoral (26\%, 21\% y $17 \%$ respectivamente), mientras que los Valles y vegas son los que concentran menor potencia instalada $(5 \%)$, (Tabla 2).

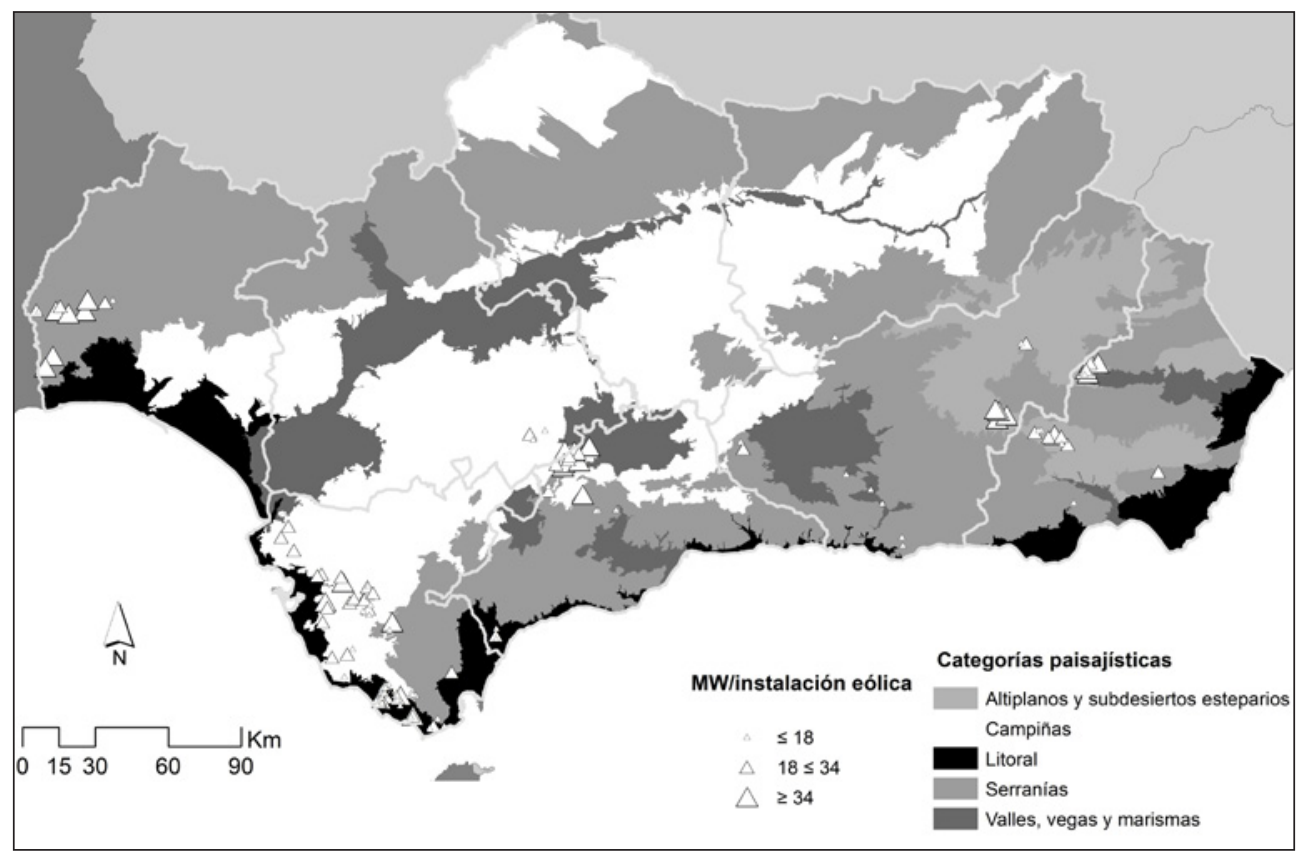

Fuente: elaboración propia.

No obstante, como se aprecia en la misma Tabla 2, esta distribución de la potencia instalada según categorías paisajísticas se modifica en el caso del número de aerogeneradores,

5 Según datos de la Agencia Andaluza de la Energía, a finales de 2012 Andalucía poseía en torno a 3252 MW de potencia eólica instalada repartidos en 144 parques eólicos diferentes. 
de manera que en esta ocasión es el Litoral junto con las Campiñas y Serranías las que aglutinan mayor número de ellos, debido a factores que se detallarán más adelante.

Tabla 2

DISTRIBUCIÓN DE LA POTENCIA EÓLICA INSTALADA EN ANDALUCÍA SEGÚN CATEGORÍAS PAISAJISTICAS 2010-2011

\begin{tabular}{|l|c|c|c|c|}
\hline \multicolumn{1}{|c|}{$\begin{array}{c}\text { CATEGORIAS } \\
\text { PAISAJISTICAS }\end{array}$} & $\begin{array}{c}\text { POTENCIA } \\
\text { INSTALADA } \\
\text { (MW) }\end{array}$ & $\begin{array}{c}\% \\
\text { POTENCIA } \\
\text { INSTALADA }\end{array}$ & No AEROG. & \% AEROG. \\
\hline LITORAL & 522,65 & 17,5 & 538 & 27,5 \\
\hline $\begin{array}{l}\text { ALTIPLANOS Y } \\
\text { SUBDESIERTOS } \\
\text { ESTEPARIOS }\end{array}$ & 632,71 & 21,1 & 313 & 16,0 \\
\hline CAMPIÑAS & 919,54 & 30,7 & 525 & 26,9 \\
\hline SERRANÍAS & 780,64 & 26,1 & 514 & 26,3 \\
\hline VALLES Y VEGAS & 136,94 & 4,6 & 64 & 3,3 \\
\hline TOTALANDALUCÍA & $\mathbf{2 9 9 2 , 4 8}$ & $\mathbf{1 0 0}$ & $\mathbf{1 . 9 5 4}$ & $\mathbf{1 0 0}$ \\
\hline
\end{tabular}

Fuente: elaboración propia.

Se observa por tanto, a raíz de lo descrito anteriormente, cómo se muestran amplias diferencias en la implantación de los parques eólicos sobre los paisajes andaluces. Ante esta distribución tan desigual se realiza a continuación un análisis más detallado de ésta, prestándose especial atención al año de instalación y el tamaño de los parques eólicos en cada ámbito paisajístico definido en el Mapa de los paisajes de Andalucía, así como a la densidad de aerogeneradores, aspectos no contemplados hasta el momento pero de gran repercusión en la mayor o menor aceptación social de éstos.

\section{IV.1. Litoral andaluz}

La presencia de instalaciones de energía eólica en esta categoría paisajística se vincula a tres de las cinco áreas paisajísticas definidas para el litoral andaluz en el Mapa de Paisajes de Andalucía (Costas con sierras litorales, Costas con campiñas costeras y Costas bajas y arenosas), todas ellas se localizan en la provincia de Cádiz (Figura 3). Se observa en la Tabla 3 como son las Costas con sierras litorales, concretamente en el ámbito paisajístico de las Sierras del Estrecho, las que aglutinan la mayor potencia instalada del litoral andaluz (en torno al $80 \%$ del total, 434,5 MW), con 464 aerogeneradores instalados.

El resto de la potencia eólica instalada en el litoral se reparte entre las Costas con campiñas costeras, concretamente en la Depresión de Jimena de la Frontera, y en las Costas bajas y arenosas, en la Bahía de Cádiz y el Litoral del Estrecho.

A pesar de constituir una de las categorías paisajísticas con mayor número de aerogeneradores instalados, con casi el $28 \%$ de los presentes en la región, que aglutinan el $17,5 \%$ de la potencia total instalada (Tabla 2), los aerogeneradores en el Litoral son por lo general de menor tamaño que la media regional. Ello se debe a que en este área se localizaron los prime- 


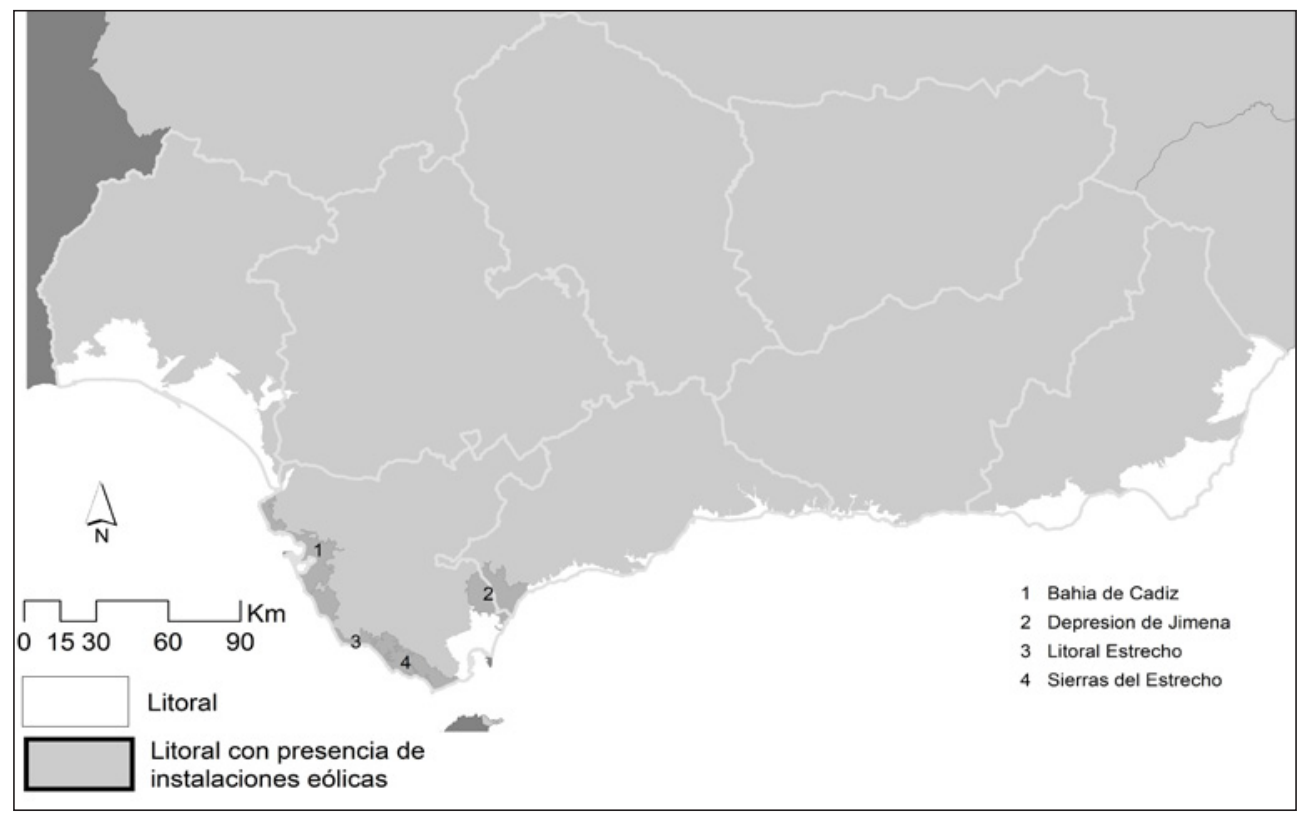

Fuente: elaboración propia.

Tabla 3

DISTRIBUCIÓN DE LA POTENCIA EÓLICA INSTALADA SOBRE EL LITORAL ANDALUZ

\begin{tabular}{|l|l|c|c|c|c|}
\hline \multirow{2}{*}{ Área paisajística } & Ámbito paisajístico & $\begin{array}{c}\text { Potencia } \\
\text { instalada } \\
\text { (MW) }\end{array}$ & $\begin{array}{c}\mathbf{N}^{\circ} \\
\text { aerog. }\end{array}$ & $\begin{array}{c}\text { MW / } \\
\text { aerog. }\end{array}$ & $\begin{array}{c}\text { Año de implantación } \\
\text { del primer parque } \\
\text { eólico }\end{array}$ \\
\hline $\begin{array}{l}\text { Costas bajas y } \\
\text { arenosas }\end{array}$ & Bahía de Cádiz & 38,95 & 20 & 1,94 & 2008 \\
\cline { 2 - 6 } & Litoral del Estrecho & 7,8 & 4 & 1,95 & 2007 \\
\hline $\begin{array}{l}\text { Costas con } \\
\text { campiñas costeras }\end{array}$ & Depresión de Jimena & 41,4 & 50 & 0,83 & 2000 \\
\hline $\begin{array}{l}\text { Costas con sierras } \\
\text { litorales }\end{array}$ & Sierras del Estrecho & 434,5 & 464 & 0,93 & 1990 \\
\hline & Litoral & $\mathbf{5 2 2 , 6 5}$ & $\mathbf{5 3 8}$ & $\mathbf{0 , 9 7}$ & $\mathbf{1 9 9 0}$ \\
\hline & Andalucía & $\mathbf{2 9 9 2 , 4 8}$ & $\mathbf{1 . 9 5 4}$ & $\mathbf{1 , 5 3}$ & $\mathbf{1 9 8 9}$ \\
\hline
\end{tabular}

Fuente: elaboración propia.

ros parques eólicos instalados en la región ${ }^{6}$ y, por tanto, las turbinas eólicas son más antiguas y pequeñas, hecho también responsable del amplio número de aerogeneradores instalados sobre esta categoría paisajística, pues al ser éstos de primera generación, se necesitaba mayor

6 A excepción del Parque eólico Monteahumada, instalado en 1989 en Los Alcornocales. 
número de ellos para conseguir una potencia instalada determinada. No obstante, en la actualidad se está procediendo al repowering de gran parte de estos parques eólicos, es decir, a la sustitución de equipos antiguos de menor potencia y eficiencia por máquinas nuevas de mayor capacidad y rendimiento, lo que ha permitido disminuir el número de aerogeneradores instalados e incrementar el aprovechamiento del recurso eólico.

\section{IV.2. Altiplanos y subdesiertos}

Los altiplanos y subdesiertos esteparios en Andalucía se localizan fundamentalmente en el sureste andaluz, en torno a la Depresión de Guadix, Hoya de Baza y en el Desierto de Tabernas (Figura 4). La presencia eólica en los Altiplanos y Subdesiertos se asocia al 21,1\% de la potencia eólica instalada en la región (Tabla 2), con 313 aerogeneradores instalados, y su localización se vincula a dos de las tres áreas paisajísticas definidas para los altiplanos y subdesiertos en la región. Las campiñas esteparias constituyen así el área paisajística que no posee presencia de instalaciones eólicas.

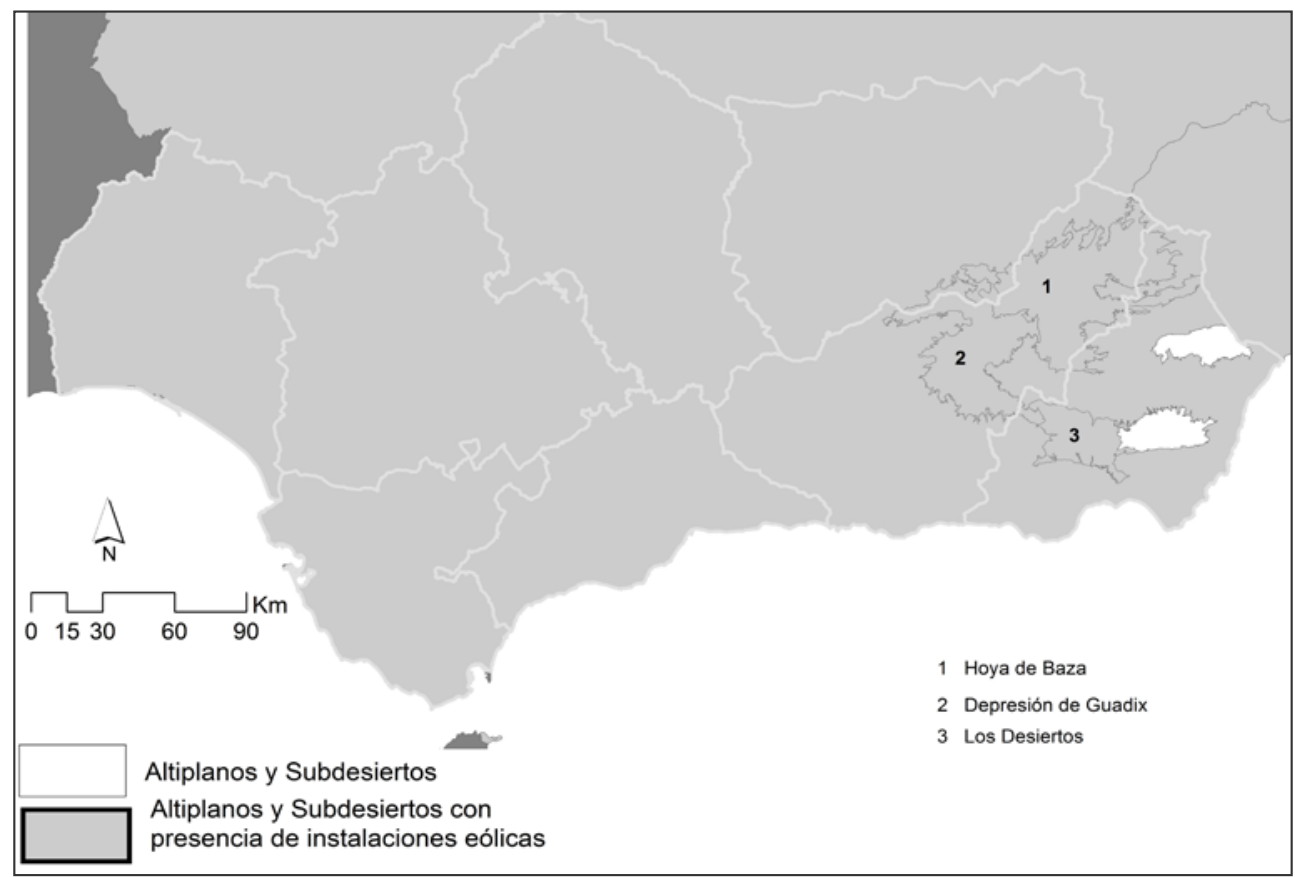

Fuente: elaboración propia.

Los primeros aerogeneradores sobre estos espacios se implantan a partir del año 2005 en la Hoya de Baza (Tabla 4) con casi 225 MW de potencia instalada, y del año 2007 sobre 
los ámbitos paisajísticos Desierto de Tabernas y Depresión de Guadix, con 126 y 282 MW respectivamente, de manera que el avance tecnológico experimentado en los últimos años por el sector ha posibilitado la implantación en este territorio de aerogeneradores con una gran potencia unitaria, por encima de los $2 \mathrm{MW}$, superando ampliamente la media regional (1,53 MW/aerog.).

Tabla 4

DISTRIBUCIÓN DE LA POTENCIA EÓLICA INSTALADA SOBRE ALTIPLANOS ESTEPARIOS Y SUBDESIERTOS ANDALUCES

\begin{tabular}{|l|l|c|c|c|c|}
\hline \multicolumn{1}{|c|}{$\begin{array}{c}\text { Área } \\
\text { paisajistica }\end{array}$} & Ámbito paisajístico & $\begin{array}{c}\text { Potencia } \\
\text { instalada } \\
(\mathbf{M W})\end{array}$ & $\begin{array}{c}\mathbf{N}^{\circ} \\
\text { aerog. }\end{array}$ & $\begin{array}{c}\text { Mw./ } \\
\text { aerog. }\end{array}$ & $\begin{array}{c}\text { Año de implantación del } \\
\text { primer parque eólico }\end{array}$ \\
\hline $\begin{array}{l}\text { Altiplanos } \\
\text { esteparios }\end{array}$ & Depresión de Guadix & 282,3 & 137 & 2,06 & 2007 \\
\cline { 2 - 6 } & Hoya de Baza & 224,58 & 112 & 2,005 & 2005 \\
\hline Subdesiertos & Desierto de Tabernas & 125,83 & 64 & 1,96 & 2007 \\
\hline & $\begin{array}{l}\text { Altiplanos y } \\
\text { Subdesiertos }\end{array}$ & $\mathbf{6 3 2 , 7 1}$ & $\mathbf{3 1 3}$ & $\mathbf{2 , 0 2}$ & $\mathbf{2 0 0 5}$ \\
\hline & Andalucía & $\mathbf{2 9 9 2 , 4 8}$ & $\mathbf{1 . 9 5 4}$ & $\mathbf{1 , 5 3}$ & $\mathbf{1 9 8 9}$ \\
\hline
\end{tabular}

Fuente: elaboración propia.

\section{IV.3. Serranías}

Por lo general las serranías andaluzas se han caracterizado tradicionalmente por la existencia de una economía de autoabastecimiento agro-silvo-pastoril, muy adaptada a las hostiles condiciones del medio para la agricultura. Ello ha hecho que estos espacios conserven por lo general una alta naturalidad, que ha derivado en la declaración de gran parte de estos territorios como espacios naturales protegidos.

La presencia eólica instalada sobre las serranías andaluzas, con 780,64 MW y 514 aerogeneradores, aparece distribuida sobre un total de 10 ámbitos paisajísticos diferentes (Figura 5 y Tabla 5), pertenecientes a dos de las tres áreas paisajísticas definidas para la categoría paisajística de Sierras: las Serranías de baja montaña y de montaña media.

La mayor potencia eólica instalada se localiza con diferencia en el Andévalo occidental, con 227 aerogeneradores y 383,6 MW instalados, seguido de la Sierra de Ronda-Grazalema y Los Alcornocales (150 y 102 MW respectivamente) y la menor en la Sierra de Baza y Filabres, con sólo un aerogenerador instalado. Por otro lado, respecto al tamaño de los aerogeneradores, es la Sierra de Alhamilla y Cabrera la que posee aerogeneradores de mayor tamaño (2,3 MW/aerog.), debido a que la mayor tardanza en su implantación en comparación con el resto de parques eólicos de las sierras (el primer aerogenerador se instala en este ámbito paisajístico en 2009), le permiten incorporar el avance tecnológico experimentado.

Por otro lado, es destacable el gran tamaño de los aerogeneradores instalados en la Sierra de Los Alcornocales, que supera la media regional, a pesar de ser el primer ámbito donde se implantan estas infraestructuras. Ello se debe, a la repotenciación de los aerogeneradores instalados. 


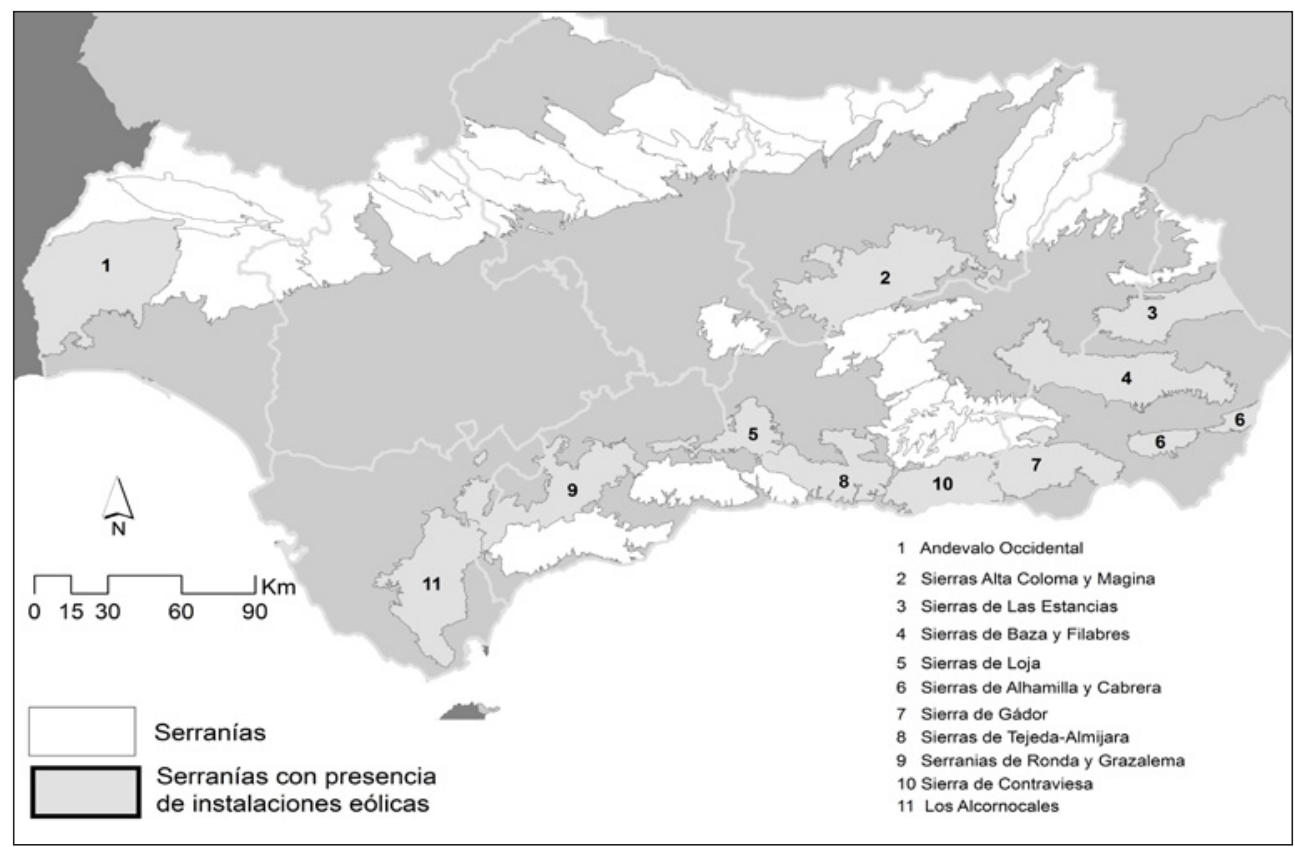

Fuente: elaboración propia.

Tabla 5

DISTRIBUCIÓN DE LA POTENCIA EÓLICA INSTALADA SOBRE LAS SERRANÍAS ANDALUZAS

\begin{tabular}{|c|c|c|c|c|c|}
\hline $\begin{array}{c}\text { Área } \\
\text { paisajística }\end{array}$ & Ámbito paisajístico & $\begin{array}{l}\text { Potencia } \\
\text { instalada } \\
\text { (MW) }\end{array}$ & $\begin{array}{c}\mathrm{N}^{0} \\
\text { aerog. }\end{array}$ & $\begin{array}{l}\text { MW/ } \\
\text { aerog. }\end{array}$ & $\begin{array}{c}\text { Año de } \\
\text { implantación del } \\
\text { primer parque } \\
\text { eólico }\end{array}$ \\
\hline \multirow{8}{*}{$\begin{array}{l}\text { Serranías de } \\
\text { montaña media }\end{array}$} & Los Alcornocales & 102,15 & 54 & 1,89 & 1989 \\
\hline & Sierra de Gádor & 13,2 & 40 & 0,33 & 1999 \\
\hline & Sierras Alta Coloma y Mágina & 15,18 & 23 & 0,66 & 2002 \\
\hline & Sierras de Loja & 35,7 & 42 & 0,85 & 2003 \\
\hline & Serranía de Ronda y Grazalema & 150,32 & 69 & 2,17 & 2005 \\
\hline & Sierras de Las Estancias & 18,01 & 9 & 2,00 & 2005 \\
\hline & Sierra de Contraviesa & 30,6 & 36 & 0,85 & 2006 \\
\hline & Sierras de Baza y Filabres & 1,98 & 1 & 1,98 & 2007 \\
\hline \multirow{4}{*}{$\begin{array}{l}\text { Serranías de } \\
\text { baja montaña }\end{array}$} & Sierras de Alhamilla y Cabrera & 29,9 & 13 & 2,30 & 2009 \\
\hline & Andévalo Occidental & 383,6 & 227 & 1,69 & 2003 \\
\hline & Sierras & 780,64 & 514 & 1,51 & 1989 \\
\hline & Andalucía & 2992,48 & 1.954 & 1,53 & 1989 \\
\hline
\end{tabular}

Fuente: elaboración propia. 


\section{IV.4. Campiñas}

Inmersas en el gran triángulo que dibuja la Depresión del Guadalquivir, las campiñas constituyen un medio muy heterogéneo y lideran la implantación eólica en la región en términos absolutos, con 920 MW (en torno al 31\% del total instalado en la región) (Tabla 2).

La presencia de instalaciones eólicas en esta categoría paisajística se localiza en dos de las tres áreas paisajísticas definidas para la misma: Campiñas alomadas, acolinadas y sobre cerros, concretamente en las Campiñas de Jerez y Sidonia, y las Campiñas de piedemonte, en el Piedemonte Subbético (Figura 6); con 96,8, 493 y 330 MW instalados respectivamente (Tabla 6).

Tal y como ocurre para los ámbitos paisajísticos anteriormente descritos, allí donde la implantación se ha producido de manera más tardía se aprovechan las mejoras y avances técnicos producidos sobre los aerogeneradores, lo que supone un mayor tamaño de éstos, superando a la media regional. Así por ejemplo, en las Campiñas de Sidonia, la energía eólica está presente desde el año 2001, mientras que para el caso del Piedemonte Subbético la implantación de los primeros parques eólicos se produce de manera más tardía, en 2007, lo que se traduce en una mayor potencia media por aerogenerador en el segundo caso.

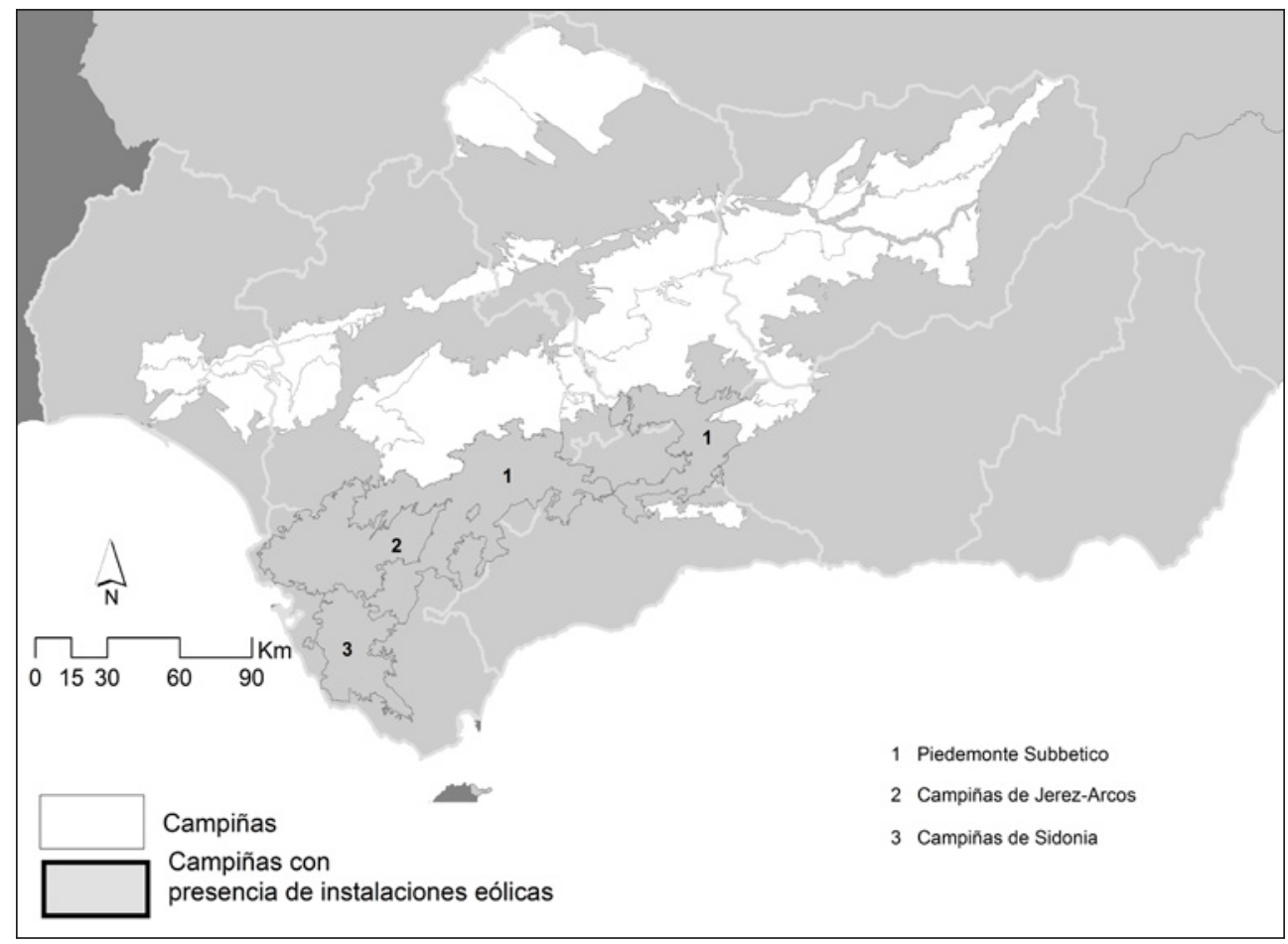

Fuente: elaboración propia. 
Tabla 6

DISTRIBUCIÓN DE LA POTENCIA EÓLICA INSTALADA SOBRE LAS CAMPIÑAS ANDALUZAS

\begin{tabular}{|l|l|c|c|c|c|}
\hline \multicolumn{1}{|c|}{ Área paisajística } & \multicolumn{1}{|c|}{$\begin{array}{c}\text { Ámbito } \\
\text { paisajístico }\end{array}$} & $\begin{array}{c}\text { Potencia } \\
\text { instalada } \\
\text { (MW) }\end{array}$ & $\begin{array}{c}\mathbf{N}^{0} \\
\text { aerog. }\end{array}$ & $\begin{array}{c}\text { Añ̃o de } \\
\text { aerog. }\end{array}$ & $\begin{array}{c}\text { implantación del } \\
\text { primer parque } \\
\text { eólico }\end{array}$ \\
\hline $\begin{array}{l}\text { Campiñas alomadas, } \\
\text { acolinadas y sobre } \\
\text { cerros }\end{array}$ & $\begin{array}{l}\text { Campiñas de } \\
\text { Jerez-Arcos }\end{array}$ & 96,8 & 63 & 1,53 & 2005 \\
\cline { 2 - 6 } & $\begin{array}{l}\text { Campiñas de } \\
\text { Sidonia }\end{array}$ & 493,05 & 292 & 1,68 & 2001 \\
\hline $\begin{array}{l}\text { Campiñas de } \\
\text { pidedemonte }\end{array}$ & $\begin{array}{l}\text { Piedemonte } \\
\text { subbético }\end{array}$ & 329,69 & 170 & 1,93 & 2007 \\
\hline & Campiñas & $\mathbf{9 1 9 , 5 4}$ & $\mathbf{5 2 5}$ & $\mathbf{1 , 7 5}$ & $\mathbf{2 0 0 1}$ \\
\hline & Andalucía & $\mathbf{2 9 9 2 , 4 8}$ & $\mathbf{1 . 9 5 4}$ & $\mathbf{1 , 5 3}$ & $\mathbf{1 9 8 9}$ \\
\hline
\end{tabular}

Fuente: elaboración propia.

\section{IV.5. Valles, vegas y marismas}

La presencia eólica sobre esta categoría paisajística se centra en los paisajes de valles y vegas, concretamente sobre Valles, vegas y marismas interiores (Depresión de Antequera y Vega de Granada) y sobre Vegas y valles intramontanos (Figura 7). Es una presencia todavía débil, si bien, aquí se encuentran los aerogeneradores de mayor tamaño de la región (en términos de potencia instalada), que surgen en los últimos años, a excepción del Valle del Lecrín, donde el primer aerogenerador se instala en 2004 (Tabla 7).

De los análisis realizados anteriormente se constatan ciertas tendencias generales en la implantación de instalaciones eólicas sobre el paisaje, de manera que esta actividad ha ido expandiendo su localización desde paisajes con una alta presencia del recurso eólico (Estrecho de Gibraltar o Sierras de media montaña), a lugares muy específicos donde las condiciones locales los hacen aptos para la instalación, como son emplazamientos concretos en los altiplanos, campiñas y subdesiertos; y, por último, a lugares donde a priori el viento resultaba difícilmente aprovechable pero donde el avance tecnológico experimentado en el sector los ha hecho viables, como son los valles y vegas. En este sentido, todo hace prever que en los próximos años los valles, vegas y marismas, junto con las campiñas y el litoral constituirán espacios de gran importancia para la implantación eólica por varios motivos.

En el caso de las campiñas y de los valles, vegas y marismas, como ya se ha mencionado con anterioridad, los avances tecnológicos experimentados por los aerogeneradores, de mayor altura y potencia (llegando a alcanzar los 2,31 MW/aerog.), permiten el aprovechamiento eólico de estas zonas, que por lo general presentan menores intensidades de viento. Ello, unido a la saturación de la red producida en otras categorías paisajísticas más tradicionales en la implantación eólica, así como a determinadas características generales existentes en estas áreas que garantizan la rentabilidad energética y económica de las instalaciones (relieve llano, alta presencia de red viaria y ausencia de grandes obstáculos que puedan provocar turbulencias en el viento), las hacen especialmente interesantes para la implantación 


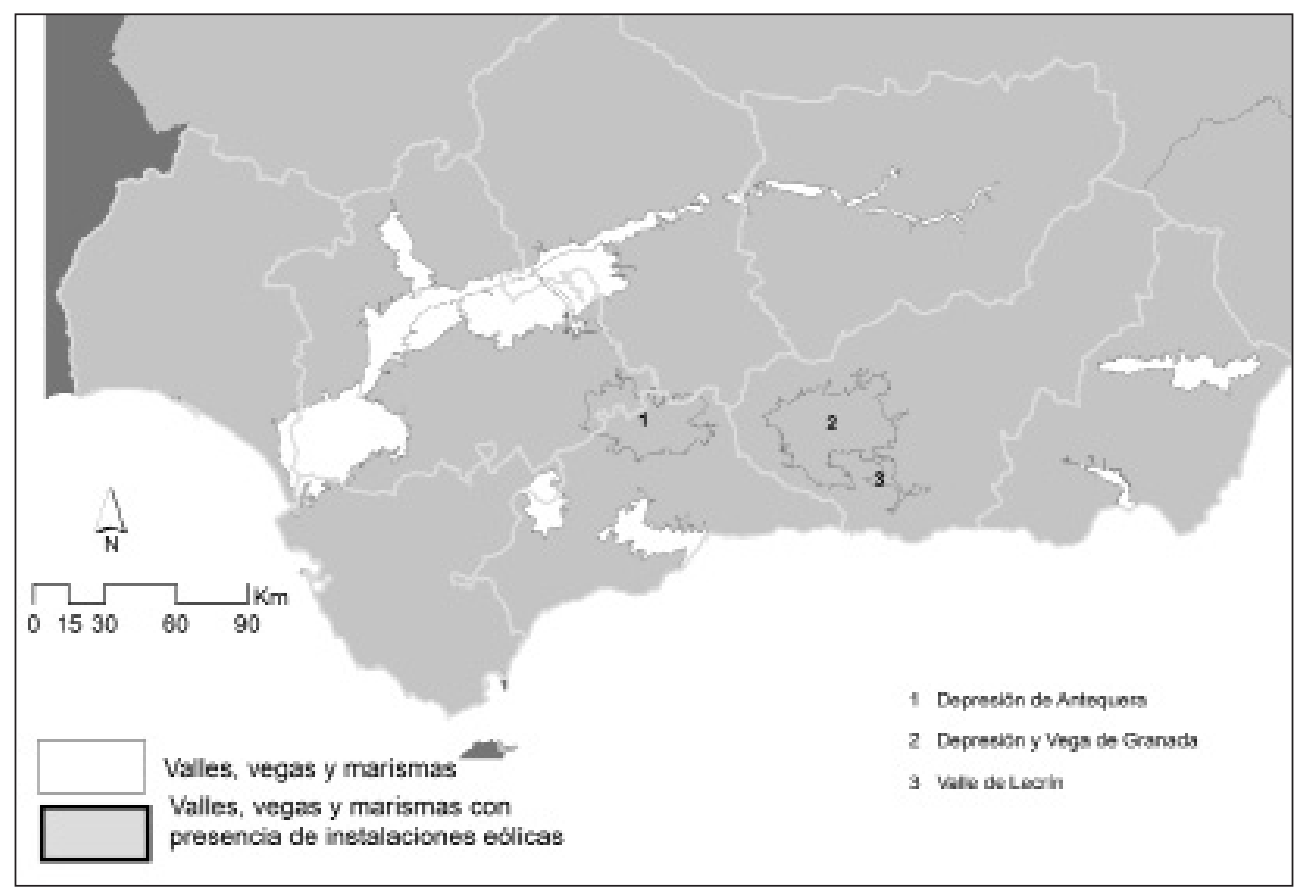

Fuente: elaboración propia.

Tabla 7

DISTRIBUCIÓN DE LA POTENCIA EÓLICA INSTALADA SOBRE LOS VALLE, VEGAS Y MARISMAS ANDALUZAS

\begin{tabular}{|l|c|c|c|c|c|}
\hline \multirow{2}{*}{ Área paisajística } & $\begin{array}{c}\text { Ámbito } \\
\text { paisajístico }\end{array}$ & $\begin{array}{c}\text { Potencia } \\
\text { instalada } \\
\text { (MW) }\end{array}$ & $\begin{array}{c}\mathbf{N}^{\circ} \\
\text { aerog. }\end{array}$ & $\begin{array}{c}\text { MW / } \\
\text { aerog. }\end{array}$ & $\begin{array}{c}\text { Año de } \\
\text { implantación del } \\
\text { primer parque } \\
\text { eólico }\end{array}$ \\
\hline $\begin{array}{l}\text { Valles, vegas } \\
\text { y marismas } \\
\text { interiores }\end{array}$ & $\begin{array}{c}\text { Depresión de } \\
\text { Antequera }\end{array}$ & 69,3 & 30 & 2,31 & 2009 \\
\cline { 2 - 6 } & $\begin{array}{c}\text { Depresión y Vega } \\
\text { de Granada }\end{array}$ & 30,3 & 14 & 2,16 & 2008 \\
\hline $\begin{array}{l}\text { Vegas y valles } \\
\text { intramontanos }\end{array}$ & $\begin{array}{c}\text { Valle de Lecrín } \\
\text { Valles, vegas y } \\
\text { marismas }\end{array}$ & 37,3 & 20 & 1,86 & 2004 \\
\hline & Andalucía & $\mathbf{2 9 9 2 , 1 8}$ & $\mathbf{1 . 9 5 4}$ & $\mathbf{1 , 5 3}$ & $\mathbf{6 4}$ \\
\hline
\end{tabular}

Fuente: elaboración propia. 
eólica. En sentido contrario, las instalaciones en dichas áreas deberán superar los problemas derivados de su alta sensibilidad para las aves, muy presentes en estas áreas paisajísticas.

En el caso del Litoral, el repowering podría producir, una vez solventados los problemas de saturación de la red eléctrica, un incremento de la potencia eólica instalada así como una disminución de la densidad de aerogeneradores en estos espacios.

\section{IDENTIFICACIÓN DE PAISAJES AFECTADOS POR INSTALACIONES DE ENERGÍA EÓLICA}

Este apartado se dirige a señalar y cuantificar aquella superficie del territorio andaluz sometida a cambios paisajísticos relevantes como consecuencia de la implantación de parques eólicos. Para ello se procederá a la integración en un SIG de los aerogeneradores existentes, digitalizados a partir de las series de ortofotografías aéreas presentes como servicio WMS en la Infraestructura de Datos Espaciales de Andalucía, según se indicó en el apartado metodológico.

Para la identificación de dichos paisajes se han seguido los criterios descritos en De Andrés e Iranzo (2012). Estos autores, en primer lugar identifican las áreas de influencia de los parques eólicos, es decir, aquellas áreas donde existan al menos tres parques eólicos (ya que la repetición de estos elementos en el paisaje juega un papel importante en la imagen global del mismo y en la percepción de la transformación de éste), y que estén separados entre sí por una distancia inferior a $15 \mathrm{~km}$; distancia elegida por dos razones:

- Porque el ojo humano difícilmente puede distinguir aerogeneradores a una distancia superior a $15 \mathrm{~km}$.

- Porque existen precedentes significativos en diferentes manuales de evaluación de los impactos paisajísticos de parques eólicos, por ejemplo en la Guide de l'Etude de l'impact sur l'environnement de parcs éoliens, publicado por el Ministerio francés de Ecología en 2010, se estipula una distancia máxima de $15 \mathrm{~km}$ como aquélla en la que el impacto visual de estas instalaciones puede llegar a ser importante.

Para este artículo, este procedimiento se ha intentado perfeccionar. En primer lugar ya que gracias a la fotointerpretación realizada se dispone de la localización exacta de cada aerogenerador, así como de la altura media de cada parque eólico; y, en segundo lugar, porque se ha tenido en cuenta la influencia del relieve en el análisis y se han identificado las áreas visibles desde cada aerogenerador.

En este sentido, se ha procedido a la realización mediante SIG de varios análisis de visibilidad desde los aerogeneradores, mediante los módulos wiewshed y visibility de Arc-Gis, teniendo en cuenta la curvatura de la Tierra, y el constructor de modelos o Model Builder.

El modelo de elevaciones utilizado es el Modelo Digital del Terreno de Andalucía, 1:10.000, generado entre 2001 y 2002 a partir de un acuerdo de colaboración entre las Consejerías de Medio Ambiente, de Agricultura y Pesca y de Obras Públicas y Transporte. Éste es en estos momentos el MDT de mayor resolución y exactitud altimétrica de la región, y está compuesto por una malla regular de puntos de 10 x 10 metros de espaciado (resolución planimétrica). 
Tras operar con el Sistema de Información Geográfica y tratar la información de las áreas de influencia (visibles hasta los $15 \mathrm{~km}$ ) de los parques eólicos en Andalucía sobre la base del Atlas de los paisajes de España, se comprueba en el análisis realizado como las implicaciones paisajísticas de la implantación eólica alcanzan más allá de la singularidad de la instalación puntual.

Así, a modo de ejemplo, estas implicaciones paisajísticas afectan al interior de algunos espacios naturales protegidos de la región donde la implantación eólica está prohibida en su normativa y, si bien no poseen ningún aerogenerador en su interior, parte de su superficie se encuentra afectada por cambios paisajísticos producidos por las implantaciones eólicas cercanas a dichos espacios. Destacan entre otros los casos del Parque Nacional de Sierra Nevada en Granada, las Reservas Naturales Laguna de Fuente de Piedra y Laguna del Gosque, en Málaga y Sevilla, el Parque Natural Sierra de Baza en Granada, y los Parajes Naturales Sierra de Alhamilla y Desierto de Tabernas en Almería (Figura 8).

Tabla 8

DISTRIBUCIÓN PROVINCIAL DE LOS PAISAJES AFECTADOS POR PARQUES EÓLICOS EN ANDALUCIAA

\begin{tabular}{|l|c|c|c|c|}
\hline PROVINCIA & $\begin{array}{c}\text { SUPERFICIE } \\
\text { AFECTADA } \\
\left(\mathrm{Km}^{2}\right)\end{array}$ & $\begin{array}{c}\text { SUPERFICIE } \\
\text { TOTAL } \\
\text { PROVINCIA } \\
\left(\mathrm{Km}^{2}\right)\end{array}$ & $\begin{array}{c}\text { \% SUPERFICIE } \\
\text { DE LA } \\
\text { PROVINCIA } \\
\text { AFECTADA }\end{array}$ & $\begin{array}{c}\text { No }^{\circ} \\
\text { AEROGENERADORES } \\
\text { INSTALADOS }\end{array}$ \\
\hline MÁLAGA & 889,10 & $7.307,40$ & 12,17 & 246 \\
\hline SEVILLA & 566,00 & $14.044,70$ & 4,03 & 59 \\
\hline ALMERÍA & 871,54 & $8.769,03$ & 9,94 & 259 \\
\hline CÁDIZ & $1.629,00$ & $7.445,18$ & 21,88 & 226 \\
\hline GRANADA & $1.883,00$ & $12.638,00$ & 14,90 & 227 \\
\hline HUELVA & 710,00 & $10.148,00$ & 6,90 & $\mathbf{1 . 9 5 4}$ \\
\hline JAÉN & 281,29 & $13.486,36$ & 2,08 & $\mathbf{7 , 8 0}$ \\
\hline $\begin{array}{l}\text { TOTAL } \\
\text { ANDALUCíA }\end{array}$ & $\mathbf{6 . 8 2 9 , 9 3}$ & $\mathbf{8 7 . 5 5 5 , 0 0}$ & & \\
\hline
\end{tabular}

Fuente: elaboración propia.

Igualmente, la localización de determinados parques eólicos sobre una provincia repercute paisajísticamente sobre los territorios de otra, hecho que ocurre también en ámbitos transfronterizos, como es el caso del límite entre Huelva y Portugal, donde los parques eólicos localizados en el Andévalo onubense producen implicaciones en determinados paisajes portugueses contiguos, que no ha sido posible evaluar por la inexistencia de un modelo digital de elevaciones portugués de características similares al utilizado en Andalucía.

Por último, atendiendo a la superficie afectada, se aprecia en la Tabla 8 como con carácter general, obviando los obstáculos derivados de la presencia de edificios y otros elementos u obstáculos que pueden hacer no visibles algunos de los aerogeneradores, $6.829,93 \mathrm{~km}^{2}$, en torno al 7,8\% de la superficie andaluza, ha sido sometido a cambios paisajísticos relevantes como consecuencia de la implantación de parques eólicos. 


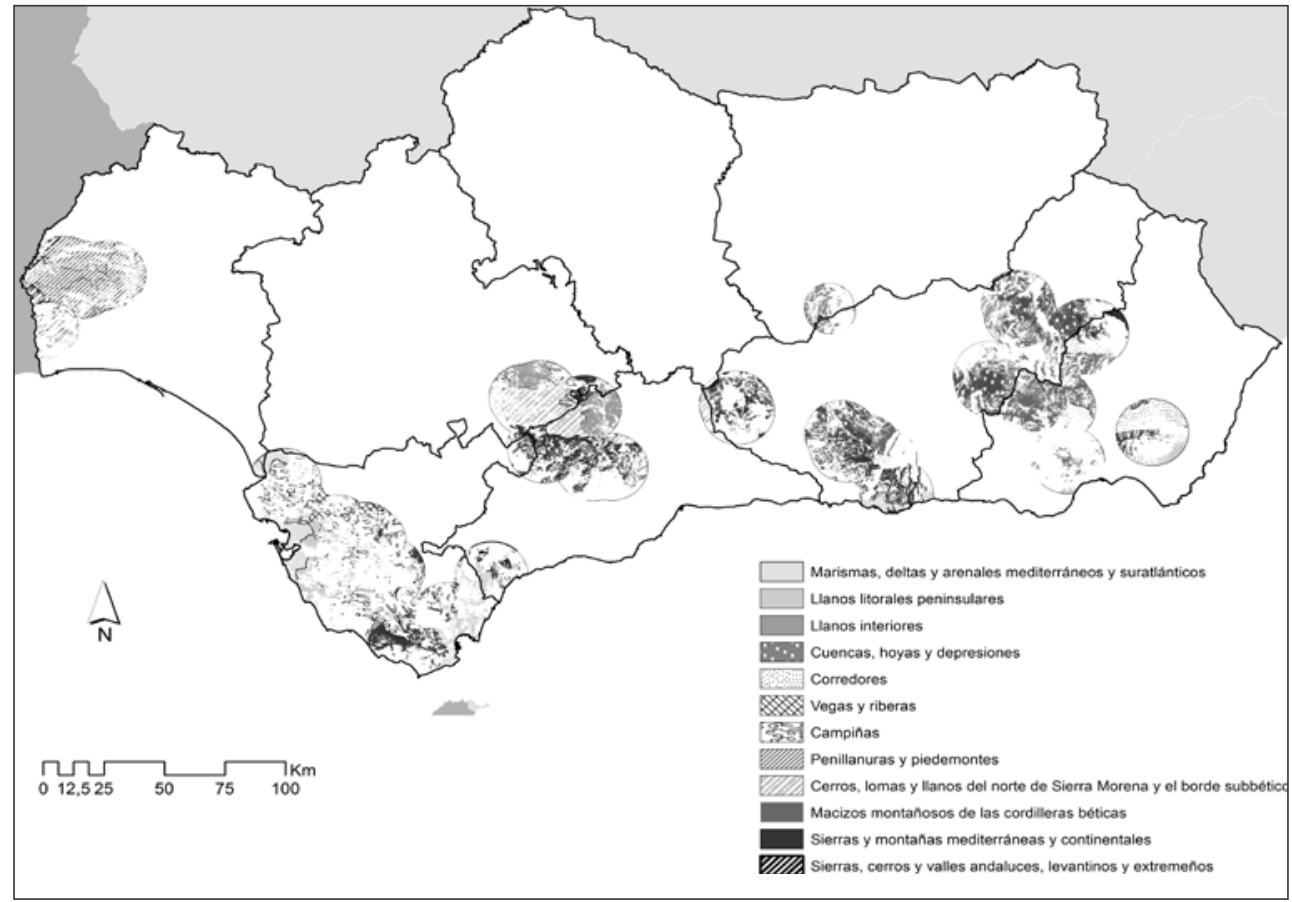

Fuente: elaboración propia.

Por provincias, Granada y Cádiz destacan como aquéllas con mayor superficie sometida a cambios paisajísticos derivados de la implantación de parques eólicos (1.883 y $1.629 \mathrm{~km}^{2}$ respectivamente), mientras que, por el contrario, Jaén y Sevilla aparecen en último lugar.

Como era previsible, las provincias con mayor número de aerogeneradores se corresponden con las que han registrado mayores cambios paisajísticos, sin embargo esta relación dista mucho de ser proporcional y así, por ejemplo, Cádiz, con 913 aerogeneradores instalados, posee una superficie sometida a cambios de este tipo menor que la provincia de Granada, con 226 aerogeneradores instalados. Ello se debe, entre otras causas vinculadas a la orografía de cada provincia, a la presencia en Cádiz de aerogeneradores de menor tamaño en cuanto a potencia y altura, así como a la gran concentración de éstos en determinados espacios ya muy saturados, en los que la cercanía entre aerogeneradores es tal que no incrementan en demasía la superficie afectada.

\section{PRINCIPALES CONFLICTOS PAISAJÍSTICOS VINCULADOS A LA ENERGÍA EÓLICA}

En Andalucía, las centrales convencionales de producción de energía se encuentran concentradas en un reducido número de emplazamientos, caracterizándolos y dándoles singularidad. Así, sólo 26 municipios de los 771 andaluces poseen alguna instalación térmica o 
hidroeléctrica (mayor de $10 \mathrm{MW}$ ), destacándose los amplios valores de potencia instalada en grupos de centrales térmicas, donde 18 de ellas aglutinan un total de $12.626 \mathrm{MW}$ de potencia instalada, mientras que las hidroeléctricas mayores de $10 \mathrm{MW}$ suman un total de $692 \mathrm{MW}$. Además de ello, Andalucía cuenta con un amplio parque de centrales de cogeneración adscritas al Régimen Especial ${ }^{7}$, que ascienden a 1.142 MW de potencia instalada

En el caso de las energías renovables, éstas presentan mayor difusión espacial que las centrales de energía convencional, de manera que contando sólo las instalaciones de energía eólica o solar, 257 municipios poseen alguna instalación de este tipo. Debido a su carácter descentralizado y disperso, las afecciones territoriales y paisajísticas de las energías renovables tienden a presentar mayor extensión que las de las energías convencionales (Baraja y Herrero, 2010), lo cual le otorga singularidad a su tratamiento.

En lo referente a los impactos ambientales, entre las metodologías para su evaluación destaca el Análisis de Ciclo de Vida (ACV), una potente herramienta que permite contabilizar el consumo de materias primas y las emisiones derivadas de cualquier actividad o proceso energético o industrial, permitiendo detectar aquellas fases que generan mayor impacto. Tal y como queda recogido en Domínguez et al. (2010), esta metodología ha sido utilizada durante los últimos años desde diferentes instituciones y empresas para determinar el impacto generado por la producción de energía eólica. Sus resultados muestran como las emisiones de $\mathrm{CO}_{2}$ producidas por las fuentes de energía renovable en general, y de energía eólica en particular, se sitúan en niveles muy inferiores de las emitidas por las tecnologías convencionales, siendo la fase de construcción la que concentra la mayor parte de las emisiones, prácticamente el $80 \%$.

Además del ACV, que permite el análisis de una actividad o proyecto a lo largo de todo su ciclo de vida, resulta necesario valorar otros impactos asociados a la actividad eólica, la mayor parte de ellos derivados del emplazamiento concreto: impacto sobre la fauna, impactos visuales y acústicos, etc.; que tendrán una gran influencia en la percepción social de éstos. Ello, ha llevado necesariamente a una atención creciente hacia los impactos paisajísticos derivados de las implantaciones eólicas; con un número cada vez más elevado de publicaciones y estudios dedicados monográficamente a analizar las relaciones entre paisaje y energías renovables (Stanton, C., 1996; Ratto, C.F. y Solari, G., 1997; Benson, J.F. et al., 2004; Baraja y Herrero, 2010; Mérida y Lobón, 2009; Prados et al., 2012; Frolova et al., 2014, etc.).

En el caso de España, la preocupación por los impactos de las energías renovables sobre el paisaje ha generado la creación de una Red española de energías renovables y paisaje, RESERP $^{8}$, que tiene por finalidad primordial «aglutinar a especialistas que muestren su

7 Definido en el artículo 27 de la Ley 54/1997, de 27 de noviembre, del Sector Eléctrico, se entiende como producción de energía en Régimen especial aquélla que se realiza en instalaciones que no superen los 50 MW, a saber: Instalaciones que utilicen la cogeneración u otras formas de producción de electricidad asociadas a actividades no eléctricas siempre que supongan un alto rendimiento energético, cuando se utilice como energía primaria alguna de las energías renovables no consumibles, biomasa o cualquier tipo de biocarburante, siempre y cuando su titular no realice actividades de producción en el Régimen ordinario o cuando se utilicen como energía primaria residuos no renovables. Se denominan de este modo en contraposición al Régimen ordinario. La actividad de generación de energía eléctrica tendrá la consideración de producción en Régimen ordinario siempre que no cumpla los requisitos exigidos para ser considerada producción en Régimen especial.

8 http://reserp.jimdo.com/ 
preocupación por una correcta inserción de las instalaciones de energías renovables en los paisajes, situar la investigación en renovables y paisaje en España a un nivel homologable con el peso internacional logrado en los desarrollos tecnológicos en el campo de las energías renovables».

Por lo que respecta a Andalucía, la implantación de parques eólicos sobre el territorio se ha realizado de manera muy rápida y ha generado una acelerada transformación de los paisajes. Este hecho coincide además con que los mejores emplazamientos para la implantación de elementos de gran tamaño individual se corresponden con los lugares de mayor exposición visual; además, la necesidad de evacuación de la energía producida está dando lugar a la proliferación de un complejo entramado de infraestructuras (infraestructura eléctrica, viaria, cimentación, plataformas e instalaciones auxiliares) que, al igual que las turbinas de viento, generan impactos sobre el territorio y el paisaje y, por tanto, deben ser incorporadas en los procesos de ordenación del territorio. A continuación se realiza un análisis individualizado de cada uno de los factores incidentes en dichos impactos paisajísticos.

\section{VI.1. Elevado ritmo de implantación de los parques eólicos}

En 2010-2011 la región andaluza poseía 129 parques eólicos que suponían un total de 2992 MW de potencia eólica instalada. La Tabla 9 identifica tres etapas de implantación de parques eólicos en Andalucía, caracterizadas por las diferentes medidas adoptadas en el contexto legislativo y planificador, tanto nacional como regional, así como por las estrategias adoptadas a nivel internacional.

A lo largo de la primera etapa, se reconoce el potencial eólico existente en algunas zonas de la región, destacando el Estrecho de Gibraltar, de forma que en esta fase todos los parques instalados se localizan en este área; si bien a partir del año 1999 la investigación de otras zonas de potencialidad lleva a la instalación de un parque en Casares (Málaga) y otro en Enix (Almería). Así, se instalan en Andalucía 12 parques eólicos (10 de ellos en Tarifa, Cádiz) con un total de 187,97 MW de potencia instalada. El ritmo de implantación en esta etapa es relativamente bajo, de $17 \mathrm{MW}$ instalados por año, debido al carácter experimental de los primeros parques unido a que, a pesar de que en 1989 se instala el primer parque eólico, no es hasta 1997, con la aprobación de la Ley 54/1997 del sector eléctrico, y el posterior desarrollo en el $R D$ 2818/1998 de 23 de diciembre, sobre producción de energía eléctrica por instalaciones abastecidas por recursos o fuentes de energías renovables cuando se establece un régimen retributivo favorable para las energías renovables, con el propósito de colocar estas energías en una posición de competencia que permita alcanzar los objetivos europeos planteados en el Libro Verde de la Energía (Comisión de las Comunidades Europeas, 2000), y lograr así que la aportación de las fuentes renovables a la demanda energética de España sea como mínimo del $12 \%$ en el año 2010 .

En la segunda etapa (2001-2006) se instalan en Andalucía un total de 1227 MW eólicos, en 58 parques eólicos. El ritmo de implantación de esta etapa se multiplica con respecto a la etapa anterior, alcanzándose los 245 MW eólicos instalados al año. Ello se debe a la aprobación del Real Decreto 436/2004, de 12 de marzo, por el que se establece el régimen económico y jurídico de la actividad de producción de energía eléctrica en Régimen Especial, que fue especialmente beneficioso para el desarrollo de la energía eólica. 
Tabla 9

CARACTERIZACIÓN DE LOS PARQUES EÓLICOS ANDALUCES SEGÚN ETAPAS DE IMPLANTACIÓN (1989-2010)

\begin{tabular}{|c|c|c|c|c|}
\hline Etapa & $\begin{array}{c}\text { Potencia } \\
\text { instalada } \\
\text { (MW) }\end{array}$ & No de parques $^{\text {MW/año }}$ & $\begin{array}{c}\text { Tamaño medio } \\
\text { (MW/parque) }\end{array}$ \\
\hline 1 $^{\text {a } \text { etapa (1989-2000) }}$ & 188 & 12 & 17 & 15,6 \\
\hline $2^{\text {a } \text { etapa (2001-2006) }}$ & 1227 & 58 & 245 & 21,15 \\
\hline $3^{\text {a etapa (2007-2010/11) }}$ & 1577 & 59 & 525 & 26,72 \\
\hline Total & $\mathbf{2 9 9 2}$ & $\mathbf{1 2 9}$ & $\mathbf{1 4 2}$ & $\mathbf{2 2 , 5 8}$ \\
\hline
\end{tabular}

Fuente: elaboración propia.

Además, en este Decreto se establecía como límite de potencia eólica a instalar en función de la capacidad de la red de transporte 13.000 MW para el territorio nacional, de manera que una vez alcanzado, se procedería a la revisión de la cuantía de las tarifas, los incentivos y las primas expresadas. Ello implica un intento por parte de las Comunidades Autónomas de aglutinar el máximo de esa potencia a instalar y se traduce en un incremento de la potencia eólica instalada de ellas.

En Andalucía, gran parte de estas instalaciones se localizan en el Estrecho de Gibraltar, y el resto se instalan en otras áreas con recurso eólico y capacidad de evacuación, delimitadas por las Zonas Eléctricas de Evacuación ${ }^{9}$. La potencia por parque eólico se incrementa en esta etapa (21,15 MW/parque), debido a la mejora tecnológica sobre los aerogeneradores (en esta etapa se alcanzan los $1500 \mathrm{~kW}$ de potencia por aerogenerador).

A lo largo de la tercera etapa (2007-2011) se instala la mayor potencia eólica en la región (1577 MW de potencia instalada repartida en 59 parques eólicos diferentes). Los parques eólicos alcanzan una potencia media de 26,72 MW/parque. Se trata de grandes parques eólicos cuya mejora tecnológica permite el aprovechamiento de espacios con menor velocidad de viento.

El ritmo de implantación en la región, 525MW/año, vuelve a multiplicarse con respecto a la etapa anterior, debido a que a lo largo de este periodo las energías renovables obtienen un gran apoyo internacional derivado del establecimiento de una política de energía para Europa (COM (07) 1final) que entre sus objetivos incluye aumentar hasta el $20 \%$ el porcentaje de las energías renovables en el consumo total en el año 2020.

En España ello se traduce en la aprobación del Real Decreto 661/2007, de 25 de mayo, por el que se regula la actividad de producción de energía eléctrica en régimen especial, que sustituye al Real Decreto 436/2004 y que establece un régimen económico que, según queda recogido en el $\mathrm{BOE}$, «protege al promotor cuando los ingresos derivados del precio del mercado fueran excesivamente bajos y elimina la prima cuando el precio del mercado es suficientemente elevado para garantizar la cobertura de sus costes». A ello se une la aprobación en Andalucía de la Ley 2/2007, de 27 de marzo, de fomento de las energías renovables y

9 ZEDES: Zonas Eléctricas de Evacuación. Establecidas mediante la Orden de 30 de septiembre de 2002 por la que se regula el procedimiento para priorizar el acceso y conexión a la red eléctrica para la evacuación de energía de las instalaciones de generación procedente de fuentes de energía renovables, residuos y cogeneración, en el caso de que las solicitudes de acceso superen la capacidad de evacuación de dichas redes. 
del ahorro y eficiencia energética de Andalucía, que establece entre sus principios generales la primacía en la producción y en la utilización de las energías renovables sobre el resto de las energías primarias; y del Plan de Sostenibilidad Energética 2007-2013, que plantea entre sus objetivos alcanzar en 2010 los 4000 MW de potencia eólica instalada (un objetivo muy amplio teniendo en cuenta que a finales de 2006 la región contaba con 1415 MW instalados). Todo ello se traduce en un beneficioso régimen de incentivos que se refleja en un incremento de la potencia eólica instalada y, por tanto, en el ritmo de implantación.

A partir de 2012 se registró cierta ralentización en la implantación eólica derivada de la entrada en vigor ese año del Real Decreto Ley 1/2012, de 27 de enero, por el que se procede a la suspensión de los procedimientos de preasignación de retribución y a la supresión de los incentivos económicos para nuevas instalaciones de producción de energía eléctrica a partir de cogeneración, fuentes de energía renovable y residuos. En este nuevo contexto, resulta necesario, realizar una revisión del sector de las energías renovables en relación a su viabilidad a corto y medio plazo y, por tanto, sobre los objetivos fijados por el Plan de Energías Renovables 2011-2020, ya aprobado, que identifica amplios objetivos para la eólica en los próximos años, ya que éstos se establecieron en una fase expansiva de la economía, cuyas normativas han sido sustituidas por otras de orientación radicalmente distinta.

En resumen, se observa como el ritmo de implantación de parques eólicos en Andalucía se ha ido intensificando con el paso de los años hasta fechas recientes, en un intento por parte de la Comunidad Autónoma de cumplir con los amplios objetivos planteados, y de los productores y empresarios de acceder a beneficiosos regímenes de retribución. En cualquier caso, este régimen de implantación tan intenso, supeditado a los amplios objetivos citados, planteados sin una valoración previa de la potencialidad del territorio andaluz para la consecución de los mismos, se traduce en el ámbito social en cierto desconcierto por parte de la población que ve incrementado en muy poco tiempo el número de instalaciones de este tipo en espacios muy concretos.

\section{VI.2. Concentración de parques eólicos}

A pesar de que por lo general las centrales de energías renovables presentan una marcada difusión espacial, cabe reflejar diferencias en la ubicación según el tipo de energía. En la Figura 9 queda patente por tanto como, en cuanto al número de instalaciones, resalta el papel claramente protagonista de las solares fotovoltaicas, que se localizan de manera dispersa a lo largo del territorio andaluz, destacando la presencia de éstas en el Valle del Guadalquivir. Sin embargo, en lo referido a la potencia instalada predomina la eólica, con más del $70 \%$ del total instalado en energías renovables.

Se observa como la actividad eólica se encuentra muy concentrada en determinadas áreas de la región, de manera que sólo 57 de los 771 municipios andaluces poseen presencia de energía eólica en su territorio. En este sentido, Tarifa, Jerez de la Frontera (ambos en Cádiz) y Serón (en Almería), son las localidades con mayor valor de potencia instalada, con 548, 248 y $208 \mathrm{MW}$ respectivamente (que suponen el 33,5\% del total instalado en la región). 
La digitalización de todos los aerogeneradores de Andalucía ha permitido el cálculo de la densidad de aerogeneradores por Kilómetro cuadrado, reflejado en la Figura 10. El municipio de Tarifa aparece con el mayor valor (con un total de 1,3 aerogeneradores $/ \mathrm{km}^{2}$ ), siendo por tanto en el que la actividad eólica ha ejercido mayor presión, superando ampliamente el valor del resto de los municipios (le siguen los municipios de Almargen (Málaga) con 0,78 , Serón y Jerez de la Frontera, ocupan ahora el tercer y cuarto lugar $(0,55$ y 0,34 respectivamente)).

Esta concentración va a ser uno de los factores esenciales a la hora de analizar y valorar los impactos paisajísticos generados por estas instalaciones en territorios concretos.

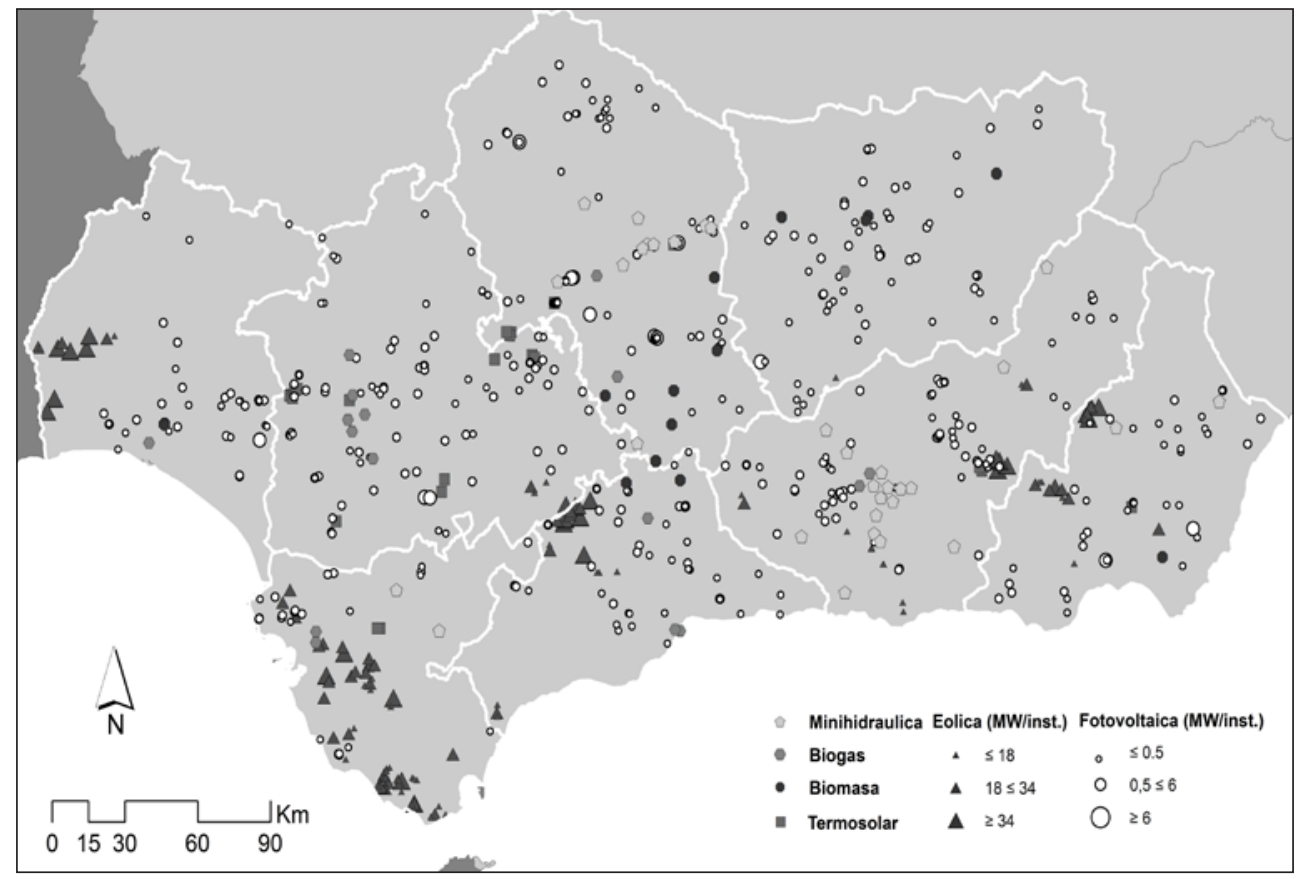

Fuente: elaboración propia.

En el caso de Tarifa, la alta concentración de aerogeneradores se ha traducido en un incremento de la presión del espacio (necesidad de tener más aerogeneradores implantados para llegar a una misma potencia eólica instalada). En este sentido, la mayor parte de los aerogeneradores instalados son obsoletos, de forma que Tarifa, que posee el $18 \%$ del total de potencia eólica instalada en la región, necesita del 28\% del total de los aerogeneradores instalados para aglutinarla; frente a otros municipios como por ejemplo Campillos, en Málaga, donde el primer parque eólico se instaló en 2008, que para reunir el 6\% de la potencia total instalada en la región necesita sólo el 3\% de los aerogeneradores instalados en Andalucía. 


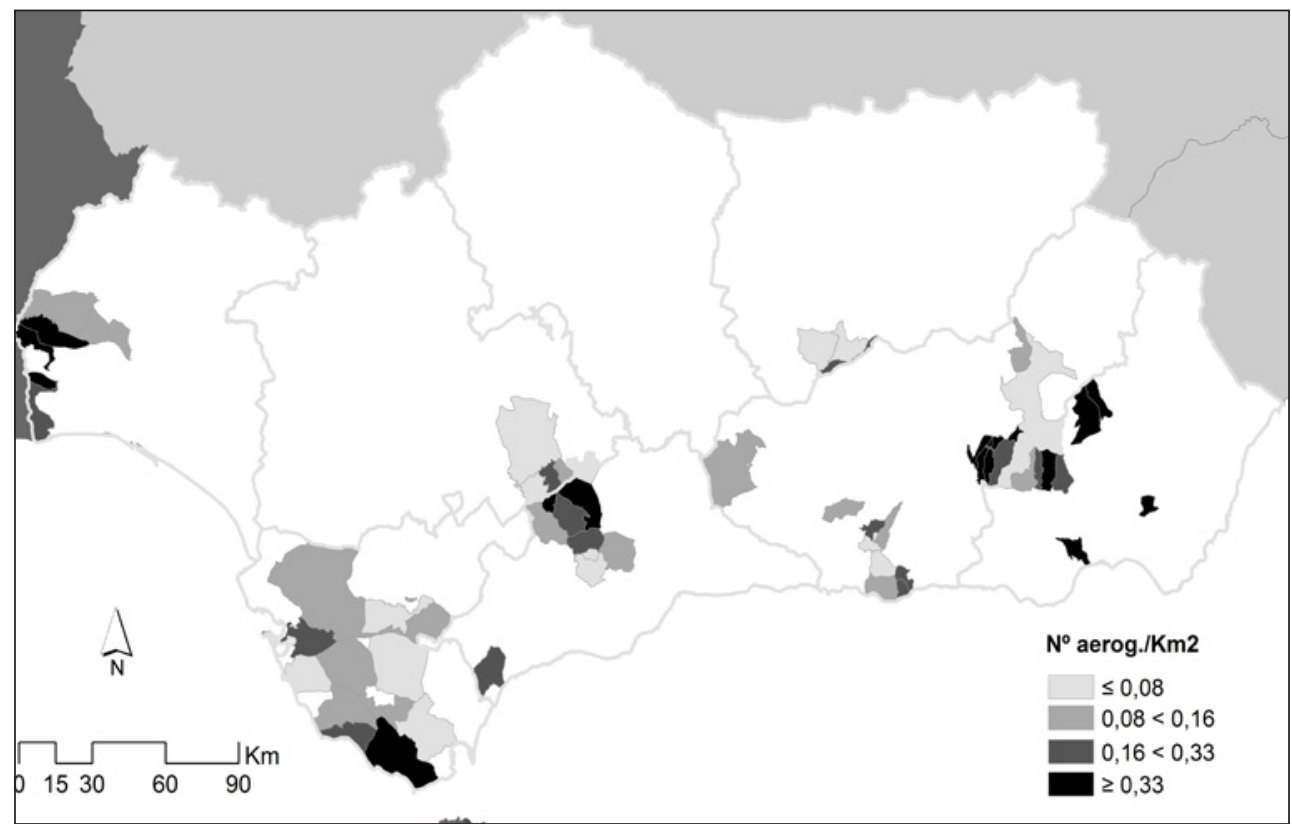

Fuente: elaboración propia.

\section{VI.3. Impactos sobre espacios con alta naturalidad}

La presencia de parques eólicos en espacios naturales protegidos o cercanos a ellos puede generar cierto rechazo por parte de la población, bien porque contradigan o deslegitimen otras líneas de actuación pública (preservación de la naturalidad) o bien por obstaculizar el desarrollo de otros usos del suelo vinculados a los valores de naturalidad que caracterizan estos espacios (atractivo turístico fundamentalmente), los cuales también han requerido importantes esfuerzos inversores y actuaciones públicas. En el caso de Andalucía 55 plantas de energía renovable (414 MW instalados) se localizan en el interior de algún espacio natural protegido de la región. De ellas, 23 se corresponden con grandes parques eólicos (Figura 11), que suman 360 aerogeneradores instalados.

En la Tabla 10 se recoge el número de aerogeneradores instalados en el interior de algún espacio natural protegido de Andalucía. Se observa como 154 aerogeneradores se localizan en el interior de algún Parque Natural, y de ellos el mayor número de ellos se localiza en el Parque Natural de Los Alcornocales, con 145 aerogeneradores instalados. El Plan de Ordenación de Recursos Naturales de dicho parque (2004) establece que será permitida la instalación de aerogeneradores para autoconsumo así como la realización de cualquier actuación que el procedimiento determine como compatible. No obstante, los aerogeneradores instalados se ubican sobre las Zonas de regulación especial, donde la actividad eólica no está prohibida. 
En el caso del parque Natural Sierra del Estrecho, con 8 aerogeneradores en su interior, el Decreto 262/2007, de 16 de octubre, por el que se aprueba el Plan Rector de Uso y Gestión del Parque Natural del Estrecho establece que en los parques eólicos presentes dentro del espacio protegido queda prohibido el aumento del número y tamaño de aerogeneradores instalados, permitiéndose sólo las actuaciones de reparación, mantenimiento o mejora de infraestructuras eólicas con objeto de minimizar su impacto ambiental. La instalación de nuevas infraestructuras para la transformación de la energía eólica no está permitida.

Tabla 10

PARQUES EÓLICOS EN ESPACIOS NATURALES PROTEGIDOS EN ANDALUCÍA

\begin{tabular}{|l|c|c|c|}
\hline \multicolumn{1}{|c|}{ Nombre espacio natural protegido } & $\begin{array}{c}\mathbf{N}^{0} \text { de aerog. } \\
\text { en ENP }\end{array}$ & $\begin{array}{c}\text { Año de } \\
\text { implanta. }\end{array}$ & $\begin{array}{c}\text { Figuras de protección } \\
\text { aplicadas al espacio }\end{array}$ \\
\hline ANDÉVALO OCCIDENTAL & 107 & $2003-2009$ & LIC \\
\hline ESTRECHO & 8 & 1996 & Parque natural. ZEPA. LIC \\
\hline LOS ALCORNOCALES & 145 & $1992-2006$ & Parque natural. ZEPA. LIC \\
\hline SIERRA DE BAZA & 42 & 2007 & Parque natural. LIC \\
\hline SIERRA DE LOJA & 17 & 2003 & LIC \\
\hline SIERRAS DE ALCAPARAÍN Y AGUAS & 40 & 1999 & LIC \\
\hline SIERRAS GÁDOR Y ÉNIX & & LIC \\
\hline
\end{tabular}

Fuente: Elaboración propia.

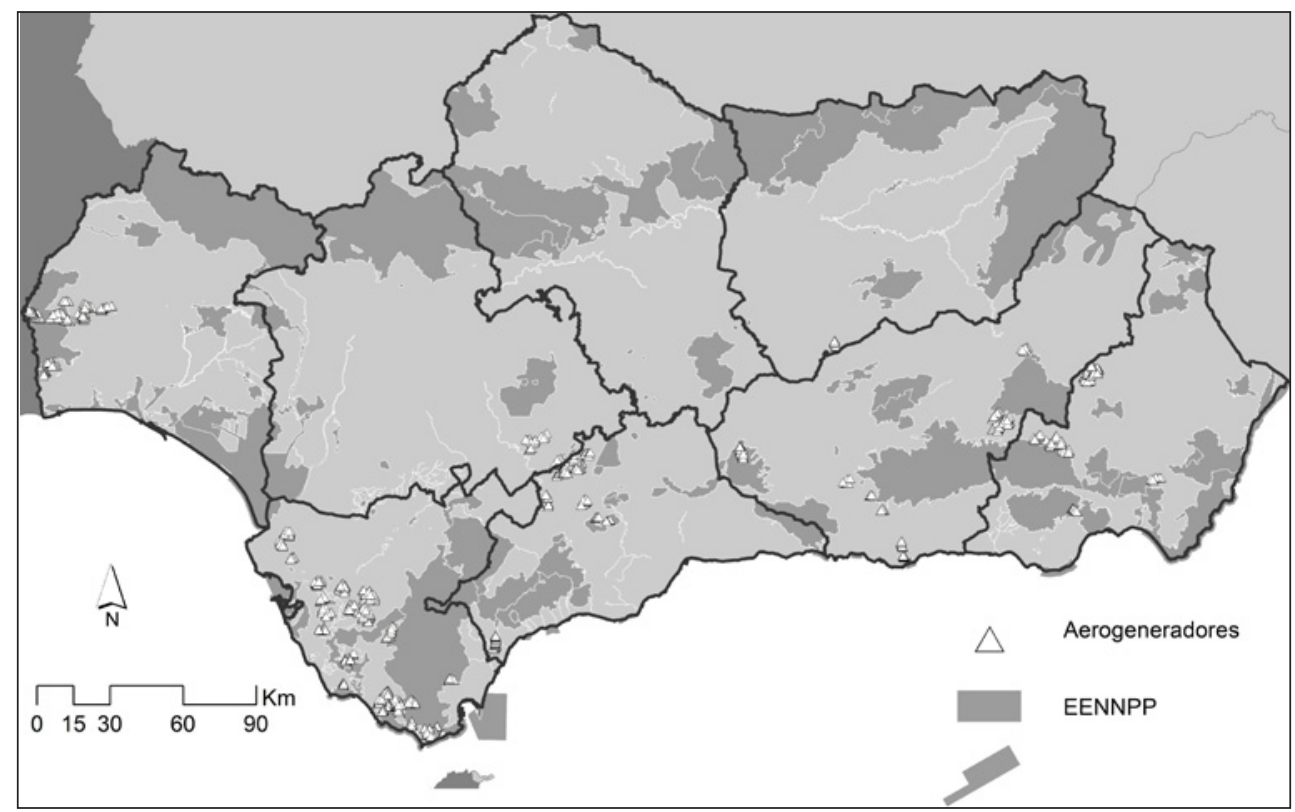

Fuente: elaboración propia. 
En el caso del Parque Natural de Sierra de Baza, el Decreto 101/2004, de 9 de marzo, por el que se aprueban el Plan de Ordenación de Recursos Naturales, establece que en las áreas calificadas como Zona A1 y Zona A2 quedan prohibidas nuevas formas de generación de energía, no estando el aerogenerador instalado en ninguna de esas zonas.

El resto de aerogeneradores, 206, se localizan en el interior de espacios catalogados como Lugares de Interés Comunitario, donde, si bien la caracterización de un espacio como LIC no excluiría inicialmente el hecho de que pudiera autorizarse un proyecto o una actividad nueva en el lugar, sí sería necesario realizar una evaluación adecuada de las repercusiones del proyecto sobre los objetivos de conservación del mismo, tal y como dispone el artículo 6.3 de la Directiva 92/43/CE del Consejo de 21 de marzo de 1992, relativa a la conservación de los hábitats naturales y de la fauna y flora silvestre que establece que: «cualquier plan o proyecto que, sin tener relación directa con la gestión del lugar [de Natura 2000] o sin ser necesario para la misma, pueda afectar de forma apreciable a los citados lugares, ya sea individualmente o en combinación con otros planes y proyectos, se someterá a una adecuada evaluación de sus repercusiones en el lugar, teniendo en cuenta los objetivos de conservación planteados»; siendo cuestionable, dado el elevado número de aerogeneradores instalados en superficies relativamente reducidas, que estas determinaciones se hayan aplicado en su plenitud.

\section{VI.4. Impacto visual}

La visibilidad de los aerogeneradores produce ciertas tensiones territoriales que superan los propios emplazamientos debido a su gran tamaño y a su localización en puntos especialmente visibles. Las turbinas pueden alcanzar una altura total de hasta 140 metros (180 metros en el caso de los modelos más recientes), siendo visibles desde 10 kilómetros de distancia en días nublados y desde distancias mucho mayores en condiciones climáticas claras.

Ante esta situación es destacable que, si bien tal y como se recoge en el Informe «Landscape and wind turbines» previamente comentado (Consejo de Europa, 2011), las turbinas eólicas individuales a menudo son vistas positivamente por los observadores, ya sean residentes o visitantes, la presencia de una alta concentración de éstas puede ocasionar impactos paisajísticos considerables.

El tratamiento del problema se complejiza si se tiene en cuenta que el impacto visual presenta dos vertientes, por un lado puede ser evaluado objetivamente, ya sea por métodos cualitativos o cuantitativos; pero por otro tiene un componente de percepción subjetiva, de tal manera que frente al mismo proyecto unas personas pueden percibirlo de manera totalmente positiva, mientras que otras pueden considerarlo negativo o molesto. Este carácter subjetivo en la percepción, implícito en toda la temática paisajística, debe ser evaluado y tenido en consideración en la fase de planificación de un parque eólico, a través de instrumentos de participación ciudadana, para así evitar futuros rechazos por parte de la población.

Por otro lado, aunque el impacto visual es muy dependiente del propio emplazamiento en sí, en la revisión bibliográfica realizada se han identificado recomendaciones respecto al diseño y características de los parques eólicos para minimizar el mismo (Hecklau, 2005; 
Stanton, 2005; Tsoutsos et al., 2006; Brusa y Lanfranconi, 2007, Domínguez Bravo et al., 2010), entre estas recomendaciones destacan la siguientes:

- Tipo y tamaño de aerogeneradores similares en un parque eólico y en los adyacentes si los hubiera.

- Aerogeneradores de colores claros (gris, beige o blanco), tripalas y con todas las palas del parque girando en el mismo sentido.

- Pocos aerogeneradores y grandes generan menor impacto que muchos y pequeños.

- Los paisajes llanos se encuadran mejor con parques eólicos en filas.

- Ubicar el parque a una cierta distancia de las viviendas habitadas.

- Instalar el tendido eléctrico subterráneo.

- Colocar luces de señalización nocturna sólo en los aerogeneradores más expuestos que bordean el perímetro del parque eólico ${ }^{10}$.

- En general, ajustar el diseño del parque de acuerdo a las peculiaridades del entorno.

\section{VI.5. Proximidad a núcleos de población}

Entre los problemas más significativos en la relación entre instalaciones eólicas y población local cabe destacar la propia proximidad física entre dichas instalaciones y los núcleos habitados. En este sentido, en la revisión bibliográfica se han encontrado diversas recomendaciones al respecto, que establecían una distancia de seguridad mínima desde 300 metros (Petit, 1994) a 500 m (Simao, 2009), con el fin de evitar por un lado el impacto sonoro de los aerogeneradores y, por otro, para proteger a la población de un posible accidente.

En este sentido, en la Tabla 11 y la Figura 12, se recoge la distancia desde los aerogeneradores a los núcleos de población de Andalucía. Se detecta como existen 30 aerogeneradores localizados a menos de $300 \mathrm{~m}$ de Facinas, núcleo de población del municipio de Tarifa. Igualmente, 26 aerogeneradores se localizan entre 301 y $500 \mathrm{~m}$ de un núcleo de población; 217 aerogeneradores se ubican entre $501 \mathrm{~m}$ y $1 \mathrm{~km}$, afectando a 6362 habitantes; y 652 aerogeneradores se localizan entre 1 y $2 \mathrm{~km}$, afectando a 49.348 habitantes. El resto de aerogeneradores se sitúan a más de $2 \mathrm{~km}$ de un núcleo de población.

Aunque el volumen de población total afectada, 62.806 habitantes, no puede ser ignorado, es cierto también que en la actualidad este tipo de problemas no es probable que se reproduzcan, dado el mayor control urbanístico y territorial existente; si bien aún pueden surgir casos puntuales de viviendas ilegalmente construidas en suelo no urbanizable, que no constituirían propiamente núcleos de población.

10 La señalización e iluminación de parques eólicos deberá realizarse atendiendo a las directrices dadas en la Guía de señalamiento e iluminación de parques eólicos, elaborada por la Agencia Estatal de Seguridad Aérea. Esta guía se establece como desarrollo del capítulo 6 del Anexo 14 de la Organización de Aviación Civil Internacional (OACI), transpuesto a la legislación española mediante el Real Decreto 862/2009, de 14 de mayo del Ministerio de la Presidencia del Gobierno, por el que se aprueban las normas técnicas de diseño y operación de aeródromos de uso público y se regula la certificación de los aeropuertos de competencia del Estado. 
Figura 12

NÚCLEOS DE POBLACIÓN CON AEROGENERADORES A MENOS DE 2 KM DE DISTANCIA EN ANDALUCÍA 2010-2011

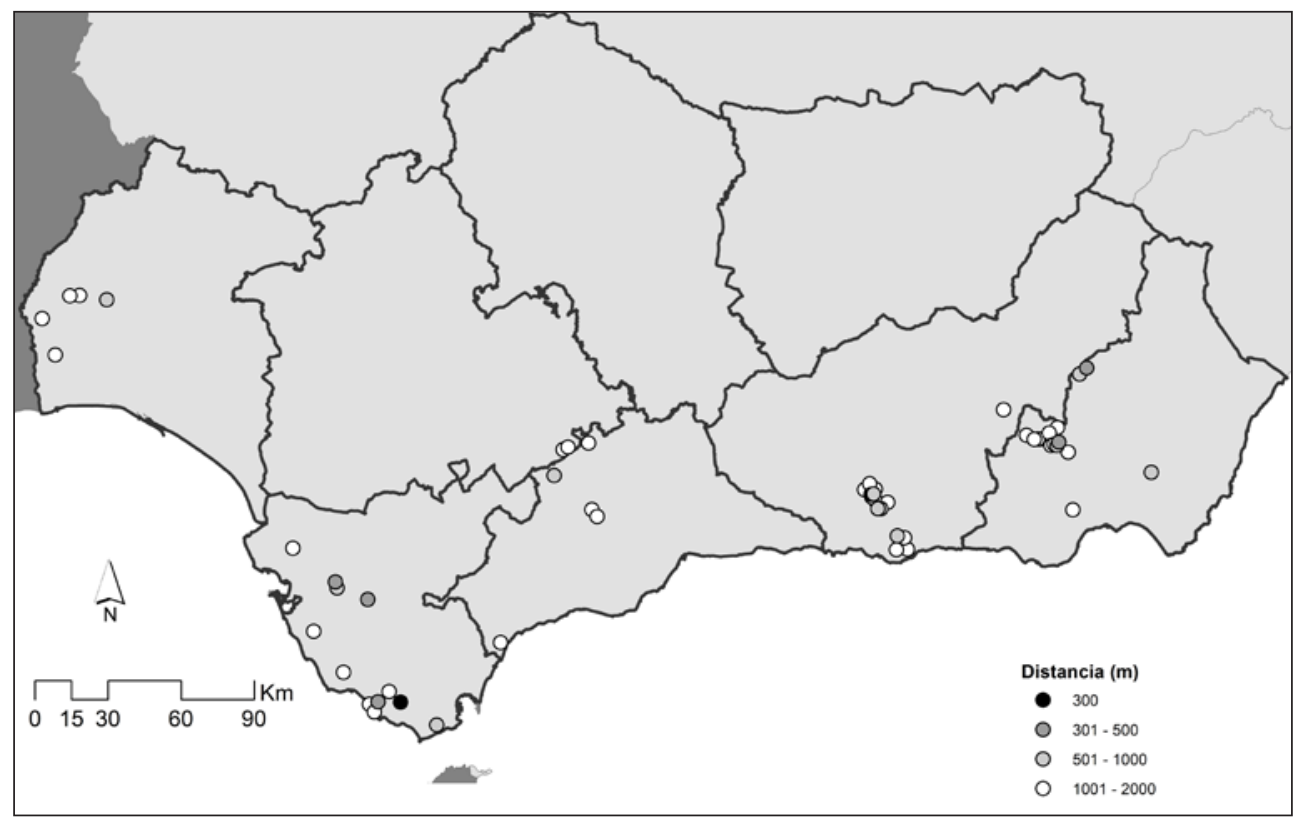

Fuente: elaboración propia.

Tabla 11

NÚMERO DE AEROGENERADORES LOCALIZADOS A MENOS DE 2 KM DE UN NÚCLEO DE POBLACIÓN Y POBLACIÓN AFECTADA

\begin{tabular}{|l|c|c|c|}
\hline Distancia $(\mathbf{m})$ & $\mathbf{N}^{0}$ aerogeneradores & Número de núcleos & Población (2012) \\
\hline$\leq 300$ & 30 & 1 & 1151 \\
\hline $301 \leq 500$ & 26 & 5 & 5945 \\
\hline $501 \leq 1000$ & 217 & 13 & 6362 \\
\hline $1001 \leq 2000$ & 652 & 39 & 49.348 \\
\hline Total & $\mathbf{9 2 5}$ & $\mathbf{5 8}$ & $\mathbf{6 2 . 8 0 6}$ \\
\hline
\end{tabular}

Fuente: elaboración propia.

\section{CONCLUSIONES}

A partir de los resultados anteriormente expuestos, las conclusiones más relevantes serían las siguientes:

- Como conclusión de carácter más general, se ha evidenciado la trascendencia de la temática tratada, la interrelación entre las instalaciones de energía eólica y el paisaje, 
dada la extensión de los territorios ya afectados, el previsible incremento de los mismos a medio-largo plazo y la intensidad de las transformaciones paisajísticas generadas. De hecho, la entrada en vigor en 2012 del RD Ley 1/2012 de 27 de enero, por el que se produce a la suspensión de nuevas instalaciones de producción de energía eléctrica a partir de fuentes de energía renovable y residuos, supone una ralentización del sector, que puede ser aprovechada como un periodo de reflexión para un análisis de implicaciones ya generadas por la rápida implantación de estas infraestructuras, así como para una revisión de los objetivos basada en una valoración real de las potencialidades del territorio andaluz para la implantación eólica. Esta reflexión deberá tener en cuenta las implicaciones paisajísticas de las futuras implantaciones que de seguro de producirán, puesto que a pesar de la ralentización derivada de la normativa estatal ya citada, los objetivos marcados por parte de la UE en energías renovables implicarán que tarde o temprano se exija al Gobierno español el establecimiento de un régimen económico que las incentive, derecho reconocido para estas instalaciones en el artículo 30.4 de la Ley del Sector Eléctrico (L54/1997).

- Desde el punto de vista metodológico, se ha confirmado la utilidad y necesidad del empleo de las Tecnologías de Información Geográfica para abordar esta temática, dadas las capacidades analíticas que ofrecen para la integración en ámbitos territoriales extensos de bases paisajísticas, por un lado, con información precisa sobre las instalaciones ubicadas en el territorio, por otro. En este sentido, se ha demostrado la necesidad de contar con una base de datos espacial de energías renovables, para la que se ha digitalizado la totalidad de los aerogeneradores existentes en Andalucía, superando anteriores procedimientos basados en tomar como referencia únicamente el centro geométrico de los parques eólicos; obteniendo así una información mucho más precisa y detallada de los impactos y efectos reales producidos por las instalaciones.

- En cuanto a la distribución de las instalaciones eólicas, se aprecia que la mayor potencia instalada se ubica en la categoría paisajística de las Campiñas (concretamente en el área paisajística de «Campiñas alomadas, acolinadas y sobre cerros»). Sin embargo, en el número de aerogeneradores predomina nítidamente la categoría Litoral (con la mayor densidad de las instalaciones en el área «Costas con sierras litorales»). En este sentido, la evolución en los últimos años muestra una modificación en las pautas de localización iniciales, pasando desde los paisajes con una alta presencia del recurso eólico (Estrecho de Gibraltar o sierras de montaña media), hacia otros espacios con condiciones locales específicas favorables a la instalación (lugares concretos con buena combinación de viento y posibilidades de evacuación de la energía en altiplanos, campiñas y subdesiertos) y, en la actualidad, gracias a la evolución tecnológica producida en los aerogeneradores, a paisajes de Valles, vegas y marismas, donde a priori el viento resultaba difícilmente aprovechable.

- Dicho desarrollo tecnológico va a implicar modificaciones trascendentes en las implicaciones territoriales y paisajísticas de estas instalaciones; así, ha supuesto la aparición de máquinas más potentes, con grandes diámetros de rotor, lo cual ha incrementado notablemente la potencia media de los parques (de 15,6 MW en la etapa inicial a 26,7 MW en la última) en paralelo a una drástica disminución del número medio de aerogeneradores por parque en Andalucía. Esta transformación reviste sustanciales consecuencias 
desde el punto de vista paisajístico, ya que los impactos negativos de estas instalaciones sobre el paisaje suelen ir vinculados a una excesiva densidad en el número de aerogeneradores, más que al efecto individual de cada uno de ellos; por lo que, en términos generales, una reducción del número de turbinas aunque su altura aumente, debería traducirse en una aminoración del impacto de los parques eólicos futuros.

- El análisis de los factores de localización predominantes para los parques eólicos existentes hasta el momento, muestra que éstos se han limitado a cuantificar la abundancia del recurso eólico y las posibilidades de evacuación de la energía generada, sin tomar en consideración los valores paisajísticos de los espacios sobre los que se asentaban y los posibles impactos que pudieran producir en los mismos. Enfoque que necesariamente deberá cambiar ante la creciente superficie afectada y la progresiva maduración de la conciencia paisajística en administraciones y población.

- La necesidad de considerar la afección visual de los parques eólicos no sólo por el lugar concreto en el que están emplazados, sino también por la más amplia extensión desde la que son percibidos, como así se ha hecho en este artículo realizando un análisis de visibilidad de cada aerogenerador gracias a la utilización de un Modelo Digital del Terreno. De esta forma se constata y cuantifica cómo las implicaciones paisajísticas alcanzan más allá de la singularidad de la instalación puntual, ejemplificándose en el caso de algunos Parques Naturales andaluces que, pese a prohibir el emplazamiento de aerogeneradores en su interior, presentan afecciones paisajísticas derivadas de la implantación de parques eólicos en su entorno inmediato.

- En relación con el análisis de visibilidad citado, cabe destacar como conclusión la amplitud de la superficie afectada, $6.829,93 \mathrm{~km}^{2}$, es decir, el 7,8\% del total de la superficie de Andalucía. Estas cifras muestran que la cuestión excede en mucho la calificación de fenómeno puntual o local, reforzando de nuevo la necesidad de establecer métodos válidos para valorar sus impactos.

- A partir de la escala regional a la que está referido este artículo, se hace necesario profundizar en estudios de detalle en la escala local, para así determinar con precisión los efectos reales. En estos estudios en detalle resultará imprescindible incluir en las metodologías instrumentos de participación pública (inviables en la escala regional), en coherencia con lo establecido en la Convención Europea del Paisaje, según la cual dicho paisaje queda definido como «cualquier parte del territorio tal como es percibida por las poblaciones», y los objetivos de calidad paisajística serán «la formulación, por parte de las autoridades públicas competentes, de las aspiraciones de las poblaciones en lo que concierne a las características paisajísticas de su entorno». De esta forma, las percepciones, valoraciones y aspiraciones de la población local deberán constituir elementos de referencia ineludibles para la toma de decisiones concretas en esta materia.

- Por último, como conclusión derivada de la coyuntura actual, es de destacar la necesidad y oportunidad de aprovechar la vigente ralentización del proceso de expansión de las instalaciones (como consecuencia de la crisis económica y de cambios normativos contrarios al mantenimiento de las ayudas públicas), como un momento de pausa que permita reflexionar sobre los impactos ya producidos, así como para desarrollar metodologías más precisas de evaluación de los efectos paisajísticos e instrumentos de actuación para prevenir su agravamiento de cara al futuro. 


\section{BIBLIOGRAFÍA}

AGENCIA ANDALUZA DE LA ENERGÍA (2012): Datos energéticos de Andalucía. Consejería de Innovación, Ciencia y Empleo. 136 pp.

AGENCIA ANDALUZA DE LA ENERGÍA (2007): Plan Andaluz de sostenibilidad energética (2007-2013). Consejería de Innovación, Ciencia y Empleo. 180 pp.

ASOCIACIÓN DE LA INDUSTRIA FOTOVOLTAICA (2007): Situación de la Energía Solar Fotovoltaica. Enlace electrónico: [www.asif.org/fi les/ASIF_Produccion_ FV_2007_Web_E2.pdf].

ASOCIACIÓN EMPRESARIAL EÓLICA: http://www.aeeolica.org/

BARAJA, E. y HERRERO, D. (2010): «Energías renovables y paisaje en Castilla y León». Nimbus, $\mathrm{n}^{\mathrm{o}}$ 25-26, pp. 21-42.

BENSON, J.F., SCOTT, K.E., ANDERSON, C., MACFARLANE, R. DUNSFORD, H. y TURMER, K. (2004): Landscape capacity study for onshore wind energy development in the western Isles. Inverness: Scotish Natural Heritage Comissioned, $\mathrm{n}^{\circ} 42$.

BRUSA, A. y LANFRANCONI, C (2007): «Guidelines for realization of wind plants and their integration in the territory». En Proceedings of EWEC 2006. Atenas, Grecia: 27 de febrero a 2 de marzo de 2007. [http://www.ewec2006proceedings.info/allfiles2/501_ Ewec2006fullpaper.pdf].

COMISIÓN DE LAS COMUNIDADES EUROPEAS (2000): Libro Verde. Hacia una estrategia europea de seguridad del abastecimiento energético. COM (2000) 769 FINAL. Bruselas. [http://europa.eu/legislation_summaries/energy/external_dimension_enlargement/ 127037_es.htm].

COMISIÓN DE LAS COMUNIDADES EUROPEAS (2007): Comunicación de la Comisión al Consejo Europeo y al Parlamento Europeo. Una política energética para Europa. COM (2007) 1 final. Bruselas. [http://eur-lex.europa.eu/LexUriServ/LexUriServ.do?uri= COM:2007:0001:FIN:ES:PDF].

CONSEJERÍA DE OBRAS PÚBLICAS Y VIVIENDA (2012): Estrategia de paisaje de Andalucía. Sevilla: Junta de Andalucía, 99 pp.

CONSEJERÍA DE OBRAS PÚBLICAS Y TRANSPORTES y CONSEJERÍA DE MEDIO AMBIENTE (2005): «Mapa de los Paisajes de Andalucía». En Atlas de Andalucía. Tomo II. Sevilla: Junta de Andalucía.

CONSEJO DE EUROPA (2000): Convenio europeo del paisaje. Florencia: 20 de noviembre de 2000. (http://conventions.coe.int/treaty/fr/Treaties/Html/176.htm).

CONSEJO DE EUROPA (2011): «Landscape and windturbines». En VI Conferencia del Consejo sobre la Convención Europea del Paisaje. Estrasburgo, 3-4 de mayo de 2011, 19 pp.

Decreto 87/2004, de 2 de marzo, por el que se aprueban el PORN y el PRUG del Parque Natural de Los Alcornocales. Boletín Oficial de Andalucía, número 88, de 5 de mayo de 2004.

Decreto 101/2004, de 9 de marzo, por el que se aprueban el Plan de Ordenación de Recursos Naturales y el Plan Rector de Uso y Gestión del Parque Natural Sierra de Baza. Boletín Oficial de la Junta de Andalucía, número 78, de 22 de abril de 2004.

Decreto 262/2007, de 16 de octubre, por el que se aprueba el Plan Rector de Uso y Gestión del Parque Natural del Estrecho y se modifica el Plan de Ordenación de los Recursos 
Naturales del Frente Litoral Algeciras-Tarifa, aprobado por Decreto 308/2002, de 23 de diciembre. Boletín Oficial de la Junta de Andalucía, n ${ }^{\circ} 233$, de 27 de noviembre de 2007.

DE ANDRÉS, C. e IRANZO, E (2012): «Desarrollo de las energías renovables y cambios paisajísticos: propuesta de tipología y localización geográfica de los paisajes energéticos de España». En Energía y territorio: dinámicas y procesos. XXII Congreso de Geógrafos Españoles. Alicante: Universidad de Alicante, pp.97-107.

DÍAZ, P., RODRÍGUEZ, J. y ZOIDO, F. (2010): «Energías Renovables y Paisaje». En IZQUIERDO, J.M. (coord.): Energías Renovables: Paisaje y Territorio Andaluz. Sevilla. Grupo de Estudios Avanzados Sobre Territorio y Medio Ambiente Textura. Volumen 1, pp. 83-101.

Directiva 92/43/CEE del Consejo, de 21 de mayo de 1992, relativa a la conservación de los hábitats naturales y de la fauna y flora silvestres. Diario Oficial de la Unión Europea, $\mathrm{n}^{\circ}$. 206 de 22 de Julio de 1992.

DOMÍNGUEZ, J., LAGO, C., PRADES, A. y DÍAZ, P. (2010): «Energías renovables y modelo energético. Una perspectiva desde la sostenibilidad». Nimbus, $\mathrm{n}^{\circ} 25-26$, pp. 42-43.

ESPEJO, C. y GARCÍA, R. (2012): «La energía eólica en la producción de electricidad en España». En Revista de Geografía Norte Grande, no 51, pp. 115-136.

FROLOVA, M., ESPEJO, C., BARAJA, E. y PRADOS, M.J. (2014): «Piasajes emergentes de las energías renovables en España». En Boletín de la Asociación de Geógrafos Españoles, n66, pp. 223-252.

FROLOVA, M. y PÉREZ, B. (2008): «El desarrollo de las energías renovables y el paisaje: Algunas bases para la implementación de la Convención europea del paisaje en la política energética española». En Cuadernos Geográficos, n 43, pp. 289-309.

HECKLAU, J. (2005): «Visual characteristics of wind turbines», En Research Meeting, diciembre de 2005. Proceedings of NWCC Technical Considerations in Siting Wind Developments [www.nationalwind.org/events/siting/proceedings.pdf].

INFRAESTRUCTURAS DE DATOS ESPACIALES DE ANDALUCÍA (http://www.ideandalucia.es/portal/web/ideandalucia/)

INSTITUTO DE ESTADÍSTICA Y CARTOGRAFÍA DE ANDALUCÍA (2013). Datos Espaciales de Andalucía Referencia Para Escalas Intermedias. [CD-ROOM]. Junta de Andalucía.

INSTITUTO DE AHORRO Y DIVERSIFICACIÓN ENERGÉTICA (2011): Plan de energías renovables (2011-2020). Madrid: Ministerio de Industria, Energía y Turismo.

MATA, R. y SANZ, C. (Dirs.), (2004): Atlas de los paisajes de España, Madrid: Ministerio de Medio Ambiente, FEDER, 683 pp.

MÉRIDA, M. y LOBÓN, R. (Coords.) (2009): Estimación de impactos propuestas de integración paisajística en las instalaciones generadoras de energía solar fotovoltaica en Andalucía. Informe Técnico. Sevilla: Consejería de Obras Públicas y Transportes. Inédito.

MINISTÉRE DE L'ECOLOGIE DU DÉVELOPPEMENT ET DE L'AMENAGEMENT DURABLES (2010): Guide de l'Etude d'impact sur l'environnement de parcs éoliens. París: Ministére de 1’Ecologie, du Développement et de 1 Aménagement Durables [http:// www.developpement-durable.gouv.fr/IMG/pdf/guide_eolien_15072010_complet.pdf.]. 
NATIONAL WIND COORDINATING COMMITTEE (1998): Permitting of Wind Energy Facilities: A Handbook. Washington, D.C.: NWCC Siting Subcommittee, 59 pp.

Ley 54/1997 del Sector Eléctrico. Boletín Oficial del Estado, n 285 , de 28 de noviembre de 1997.

Ley 2/2007, de 27 de marzo de fomento de las energías renovables y de ahorro y eficiencia energética de Andalucía. Boletín Oficial de la Junta de Andalucía n ${ }^{\circ} 109$, de 7 de mayo de 2007.

Ley Orgánica 2/2007, de 19 de marzo, de reforma del Estatuto de Autonomía para Andalucía. Boletín Oficial de Andalucía, número 56, de 20 de marzo de 2007.

ORDEN de 30 de septiembre de 2002, por la que se regula el procedimiento para priorizar el acceso y conexión a la red eléctrica para evacuación de energía de las instalaciones de generación contempladas en el Real Decreto 2818/1998, sobre producción de energía eléctrica por instalaciones abastecidas por recursos o fuentes de energías renovables, residuos y cogeneración. En Boletín Oficial de la Junta de Andalucía, número 124, 24 de octubre de 2002.

PRADOS, M.J., BARAJA, E., FROLOVA, M. y ESPEJO, C. (2012): «Integración paisajística y territorial de las energías renovables». En Ciudad y Territorio, pp.127-143.

PETIT, C. (1995): «Winds of change. GIS helps site wind farms in France. Godmanchester (Huntingdon)». GeoTecMedia, pp.17-18.

RATTO, C.F. y SOLARI, G. (1997): Wind energy and landscape. Genova, Italy: Proceedings of the international workshop WEL, 26-27 de Junio 1997.

Real Decreto 862/2009, de 14 de mayo del Ministerio de la Presidencia del Gobierno, por el que se aprueban las normas técnicas de diseño y operación de aeródromos de uso público y se regula la certificación de los aeropuertos de competencia del Estado. Boletín Oficial del Estado, número 132, de 1 de junio de 2009.

Real Decreto 2818/1998 de 23 de diciembre, sobre producción de energía eléctrica por instalaciones abastecidas por recursos o fuentes de energías renovables, residuos y cogeneración. Boletín Oficial del Estado, número 312, de 30 de diciembre de 1998.

Real Decreto 436/2004, de 12 de marzo, por el que se establece la metodología para la actualización y sistematización del régimen jurídico y económico de la actividad de producción de energía eléctrica en régimen especial. Boletín Oficial del Estado, número 75, de 27 marzo de 2004.

Real Decreto 661/2007 de 25 de mayo, por el que se regula la actividad de producción de energía eléctrica en régimen especial, que deroga el RD 436/2004. Boletín Oficial del Estado, número 126, de 26 de mayo de 2007.

Real Decreto-Ley 1/2012 de 27 de enero, por el que se procede a la suspensión de los procedimientos de preasignación de retribución y a la supresión de los incentivos económicos para nuevas instalaciones de producción de energía eléctrica a partir de cogeneración, fuentes de energía renovables y residuos. Boletín Oficial del Estado, n 24, de 28 de enero de 2012.

RED DE ESPAÑOLA DE ENERGÍAS RENOVABLES Y PAISAJE (http://reserp.jimdo. $\mathrm{com} /$ )

STANTON, C. (2005): «Visual Analysis of Windfarms Good Practice Guidance». En Technical Considerations in Siting Wind Developments: Research Meeting, United States: National Wind Coordinating Collaborative. 
STANTON, C. (1996): The Impact and Visual Design of Windfarms. Edinburgh: School of Landscape Architecture, Edinburgh College of Art.

SIMAO, A., DENSHAM, P.J., y HAKLAY, M.M. (2009): «Web-based GIS for Collaborative Planning and Public Participation: An Application to the Strategic Planning of Wind Farm Sites». En Journal of Environmental Management, n90, Issue 6, pp. 2027-2040.

TSOUTSOS, T., GOUSKOS, Z.,KARTERAKIS, E. y PEROULAKI, E (2006): «Aesthetic impact from wind parks». En European Wind Energy Conference EWEC. Athens [http:// www.ewec2006proceedings.info/allfi les2/805_Ewec2006fullpaper.pdf.]. 